УДК 519.6

MSC 65K05, 49M05

\title{
METHODS FOR PROBLEMS OF VECTOR GENERALIZED OPTIMAL CONTROL OF SYSTEMS WITH DISTRIBUTED PARAMETERS
}

\author{
O. S. Kharkov, Ya. I. Vedel, V. V. Semenov
}

Faculty of Computer Science and Cybernetics, Taras Shevchenko National University of Kyiv, Kyiv, Ukraine, E-mail: \{olehharek, yana.vedel, volodya.semenov\}@gmail.com

\section{МЕТОДИ ДЛЯ ЗАДАЧ ВЕКТОРНОГО УЗАГАЛЬНЕНОГО ОПТИМАЛЬНОГО КЕРУВАННЯ СИСТЕМАМИ 3 РОЗПОДІЛЕНИМИ ПАРАМЕТРАМИ}

\author{
О. С. Харьков, Я. І. ВеДЕЛЬ, В. В. Семенов
}

Факультет комп'ютерних наук та кібернетики, Київський національний університет імені Тараса Шевченка, Київ, Україна, E-mail: olehharek@gmail.com, yana.vedel@gmail.com, volodya.semenov@gmail.com

ABSTRACT. The paper develops the theory of existence and necessary optimality conditions for optimal control problems with a vector quality criterion for systems with distributed parameters and generalized impacts. The concept of $(K, e, \varepsilon)$-approximate efficiency is investigated. Necessary conditions for $(K, e, \varepsilon)$-approximate efficiency of admissible controls in the form of variational inclusions are proved. Methods for solving problems of vector optimization of linear distributed systems with generalized control are proposed. Convergence of algorithms with errors is proved.

KEYWORDS: vector optimization, distributed parameters system, generalized control, optimality conditions, algorithm, convergence.

АнотАція. У роботі розвинена теорія існування та необхідних умов оптимальності для задач оптимального керування з векторним критерієм якості системами, що описуються рівняннями математичної фізики з узагальненими впливами. Досліджено поняття $(K, e, \varepsilon)$-апроксимаційної ефективності. Доведено необхідні умови $(K, e, \varepsilon)$-апроксимаційної ефективності допустимих керувань у вигляді варіаційних включень. Запропоновано методи розв'язання задач векторної оптимізації лінійних розподілених систем з узагальненим керуванням. Доведено збіжність алгоритмів із похибками в ітераційних підзадачах.

КлЮчові СловА: векторна оптимізація, система з розподіленими параметрами, узагальнене керування, умови оптимальності, алгоритм, збіжність. 


\section{ВСТуП}

Теорія задач багатокритеріальної (векторної) оптимізації є одним із тих розділів сучасної теорії екстремальних задач, який інтенсивно розвивається в останні десятиліття [1-6]. Дослідженню проблем векторної оптимізації та пошуку ефективних методів їх розв'язання присвячено надзвичайно багато літератури. Мабуть, вперше математичну проблему векторної оптимізації було поставлено італійським економістом Вілфредо Парето (1848-1923). Не випадково саме в рамках математичної економіки сформулювалося одне 3 ключових для проблеми багатокритеріального вибору поняття Паретооптимальності, яке лежить в основі сучасних уявлень про кооперативну економічну ефективність.

Основні зусилля вчених концентруються на розробці таких традиційних для теорії оптимізації напрямків, як теореми існування оптимальних розв'язків, умови оптимальності, двоїстість в задачах векторної оптимізації, стійкість оптимальних розв'язків. Значну частину досліджень присвячено проблемі скаляризації, що можна пояснити тим, що зведення задачі векторної оптимізації до задачі або родини задач скалярної оптимізації відкриває шлях для використання у векторній оптимізації арсеналу добре розроблених аналітичних і чисельних методів скалярної оптимізації. Відмітимо статтю [7], де автори виклали плідну ідею побудови алгоритмів для задач пошуку оптимуму за Парето для гладких вектор-функцій.

Задачам оптимального керування зосередженими та розподіленими системами з векторними критеріями присвячено роботи $[1,4,8-14]$. У роботах [9-11] досліджувались задачі пошуку рівноваги за Нешем, а в [12-14] лінійно-квадратичні задачі Парето-оптимізації. Окремо відмітимо роботу А. Бенсусана, Ж.-Л. Ліонса, Р. Темама [15], яка містить ряд принципових постановок задач та підходів до їх розв'язання.

Мета роботи - розвинути теорію існування та необхідних умов оптимальності для задач оптимального керування з векторним критерієм якості системами, що описуються рівняннями математичної фізики з узагальненими впливами $[16,17]$. Увагу приділено побудові і дослідженню збіжності методів розв'язання задач векторної оптимізації лінійних розподілених систем. Досліджено поняття $(K, e, \varepsilon)$-апроксимаційної ефективності. Грунтуючись на векторному варіанті класичного варіаційного принципу Екланда, доведено умови $(K, e, \varepsilon)$-апроксимаційної ефективності допустимих керувань у вигляді варіаційних включень. Запропоновано методи розв'язання задач векторної оптимізації лінійних розподілених систем з узагальненим керуванням. Доведено збіжність алгоритмів із похибками в ітераційних підзадачах. Деякі з результатів були раніше опубліковані у статтях $[18,19]$.

\section{1. ПОСТАНОВКА ЗАДАЧ ТА ДОПОМІЖНІ РЕЗУЛЬТАТИ}

Нехай $W_{1}, W_{2}, H_{1}, H_{2}$ і $H-$ п'ять просторів Гільберта над полем $\mathbb{R}$. Позначимо відповідно через $\|\cdot\|_{W_{1}},\|\cdot\|_{W_{2}},\|\cdot\|_{H_{1}},\|\cdot\|_{H_{2}},\|\cdot\|_{H}$ норми в $W_{1}$, $W_{2}, H_{1}, H_{2}$ і $H$, а через $(\cdot, \cdot)_{W_{1}},(\cdot, \cdot)_{W_{2}},(\cdot, \cdot)_{H_{1}},(\cdot, \cdot)_{H_{2}},(\cdot, \cdot)_{H}-$ відповідні скалярні добутки. Нехай 
- вкладення $W_{i}$ в $H_{i}, H_{i}$ в $H$ неперервні та щільні;

- простір $H_{i}$ проміжний між $W_{i}$ та $H, i=\overline{1,2}$.

Ототожнимо простір $H$ зі спряженим до нього простором. Нехай $W_{1}^{-}$, $W_{2}^{-}, H_{1}^{-}$і $H_{2}^{-}$- простори, спряжені відповідно до $W_{1}, W_{2}, H_{1}$ і $H_{2}$. Тоді $H$ можна ототожнити 3 деякими підпросторами в $W_{1}^{-}, W_{2}^{-}, H_{1}^{-}$i $H_{2}^{-}$. Приходимо до двох ланцюжків гільбертових оснащень

$$
W_{1} \subseteq H_{1} \subseteq H \subseteq H_{1}^{-} \subseteq W_{1}^{-}, \quad W_{2} \subseteq H_{2} \subseteq H \subseteq H_{2}^{-} \subseteq W_{2}^{-},
$$

причому кожен простір щільний в наступному і вкладення неперервні.

Нехай функціонування деякої системи описується відомим нам лінійним та неперервним оператором $\mathcal{L} \in L\left(W_{1}, W_{2}^{-}\right)$і задані простір керувань гільбертів простір $V$, ототожнений зі своїм спряженим, і відображення (нелінійне) $F: V \rightarrow W_{2}^{-}$.

Через $\mathcal{L}^{+}$позначимо $H$-спряжений до $\mathcal{L}$ оператор, тобто $\mathcal{L}^{+} \in L\left(W_{2}, W_{1}^{-}\right)$ i $\langle\mathcal{L} y, p\rangle_{W_{2}^{-}, W_{2}}=\left\langle y, \mathcal{L}^{+} p\right\rangle_{W_{1}, W_{1}^{-}}$для $y \in W_{1}, p \in W_{2}$.

Для кожного керування $u \in V$ стан системи $y=y(u)$ визначається як узагальнений розв'язок операторного рівняння

$$
\mathcal{L} y=F(u),
$$

тобто, $y \in H_{1}$ - елемент, що задовольняє тотожність

$$
\left\langle y, \mathcal{L}^{+} p\right\rangle_{H_{1}, H_{1}^{-}}=\langle F(u), p\rangle_{W_{2}^{-}, W_{2}} \quad \forall p \in W_{2}: \quad \mathcal{L}^{+} p \in H_{1}^{-} .
$$

Припустимо, що виконуються апріорні оцінки

$$
\begin{gathered}
\|y\|_{H_{1}} \leq c\|\mathcal{L} y\|_{W_{2}^{-}} \quad \forall y \in W_{1}, \\
\|p\|_{H_{2}} \leq c\left\|\mathcal{L}^{+} p\right\|_{W_{1}^{-}} \quad \forall p \in W_{2} .
\end{gathered}
$$

Має місце наступна теорема [16].

Теорема 1. Якщо справджуються апріорні очінки (2), (3), то для довільного $f \in H_{2}^{-}$існуе єдиний розв'язок $y \in W_{1}$ операторного рівняння

$$
\mathcal{L} y=f,
$$

а для $f \in W_{2}^{-}$існуе единий узагальнений розв'язок $y \in H_{1}$ рівняння (4). При изому оператор $f \mapsto y$ неперервний у відповідних топологіях.

Аналогічний факт має місце і для спряженого рівняння $\mathcal{L}^{+} p=g$. Доведення апріорних оцінок вигляду (2), (3) для багатьох класів диференціальних операторів наведено в [16,20].

Справедливі твердження [16,20]: звичайний (класичний) розв'язок рівняння (4) є узагальненим; якщо узагальнений розв'язок $y \in H_{1}$ рівняння (4) належить простору $W_{1}$, то він є звичайним розв'язком; якщо $y \in H_{1}$ - узагальнений розв'язок рівняння (4) із правою частиною $f \in R(\mathcal{L})$, то $y \in W_{1}$ і є звичайним розв'язком.

Згідно теореми 1 рівняння (1) однозначно визначає стан системи $y(u)$. Крім того, нехай задано спостереження $z_{i}(u)=C_{i} y(u), i=\overline{1, m}$, де $C_{i} \in$ $L\left(H_{1}, E_{i}\right), E_{i}$ - деякі гільбертові простори, канонічно ототожнені зі своїми спряженими. 
Кожному керуванню $u \in V$ відповідає значення векторного критерію якості

$$
J(u)=\left(\begin{array}{c}
\frac{1}{2}\left\|C_{1} y(u)-a_{1}\right\|_{E_{1}}^{2} \\
\cdots \\
\frac{1}{2}\left\|C_{m} y(u)-a_{m}\right\|_{E_{m}}^{2}
\end{array}\right),
$$

де $a_{i} \in E_{i}, i=\overline{1, m}$ - задані елементи.

Припустимо, що у просторі $\mathbb{R}^{m}$ задано гострий замкнений опуклий конус $K$ з непорожньою внутрішністю, тобто множину, що задовольняе такі умови:

- $-K \bigcap K=\{0\}$ (гострота);

- $\lambda K \subseteq K(\lambda \geq 0)$ (конус);

- $K+K \subseteq K$ (опуклість конуса);

- $\operatorname{cl} K=K, \operatorname{int} K \neq \emptyset$.

Позначимо $K^{*}=\left\{k^{*} \in \mathbb{R}^{m}:\left(k^{*}, k\right)_{\mathbb{R}^{m}} \geq 0 \forall k \in K\right\}$ - невід'ємний спряжений до $K$ конус, причому int $K^{*} \neq \emptyset$ завдяки гостроті конуса $K$ [21].

Нехай у просторі $V$ задано множину допустимих керувань $U \subseteq V$.

Задача полягає у пошуку допустимих керувань $u \in U$ таких, що

$$
J(v) \notin J(u)-(K \backslash\{0\}) \quad \forall v \in U .
$$

Допустимі керування, що задовольняють (5), називаємо ефективними або оптимальними за Парето [6], а множину таких керувань позначимо $E_{K}(U)$. Задачу знаходження елементів $E_{K}(U)$ будемо позначати

$$
\begin{gathered}
J(u) \rightarrow K \text { - min, } \\
\mathcal{L} y=F(u), u \in U .
\end{gathered}
$$

Крім знаходження елементів множини $E_{K}(U)$, будемо розглядати класичні задачі пошуку строго та слабко ефективних (оптимальних за Слейтером) допустимих керувань [6], визначених відповідно так:

$$
\begin{gathered}
u \in S E_{K}(U) \Leftrightarrow J(v) \notin J(u)-K \quad \forall v \in U \backslash\{u\}, \\
u \in W E_{K}(U) \Leftrightarrow J(v) \notin J(u)-\operatorname{int} K \quad \forall v \in U .
\end{gathered}
$$

Задачі знаходження елементів множин $S E_{K}(U)$ і $W E_{K}(U)$ будемо відповідно позначати таким чином

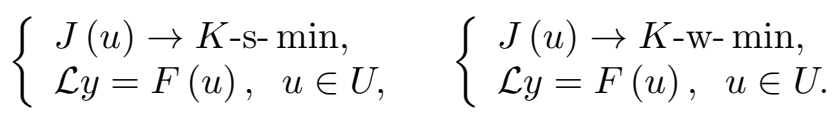

Якщо у формулах (5)-(7) замість множини $U$ поставити перетин $U \cap$ $O(u)$, де $O(u)$ - деякий окіл точки $u$, то допустиме керування $u$ будемо називати відповідно локально ефективним, локально строго ефективним і локально слабко ефективним. Позначимо відповідні множини $\operatorname{loc} E_{K}(U)$, $\operatorname{loc} S E_{K}(U)$ i $\operatorname{loc} W E_{K}(U)$.

Очевидно, що

$$
S E_{K}(U) \subseteq E_{K}(U) \subseteq W E_{K}(U)
$$

та

$$
\operatorname{loc} S E_{K}(U) \subseteq \operatorname{loc} E_{K}(U) \subseteq \operatorname{loc} W E_{K}(U)
$$


Отже, якщо $S E_{K}(U) \neq \emptyset$, то непорожні й інші з описаних тут ефективних множин, а необхідна умова локальної слабкої ефективності буде необхідною умовою локальної ефективності та локальної строгої ефективності.

\section{2. ТЕОРЕМИ ІСНУВАННЯ ТА АПРОКСИМАЦІЇ}

Використавши класичний принцип «компактність + (напів) неперервність $=$ існування», одержимо такі результати.

Теорема 2. Нехай виконано умови:

1) $\mathbb{R}_{+}^{m} \cap \operatorname{int} K^{*} \neq \emptyset$;

2) $U$ - слабко компактна підмножина простору Гільберта $V$;

3) $F: V \rightarrow W_{2}^{-}$- слабко неперервний оператор (тобто, $F: V \rightarrow W_{2}^{-}$ неперервний як оператор, діючий із простору $V$ з слабкою топологією у простір $W_{2}^{-}$також з слабкою топологією).

Тоді множина ефективних керувань $E_{K}(U)$ непорожня.

Доведення. Використаємо класичний у багатокритеріальній оптимізації метод скаляризації за допомогою лінійної згортки [6]. 3 1) випливає існування такого вектору $k^{*} \in \mathbb{R}_{+}^{m}=\left\{k=\left(k_{1}, \ldots, k_{m}\right) \in \mathbb{R}^{m}: k_{1} \geq 0, \ldots, k_{m} \geq 0\right\}$, що

$$
\left(k^{*}, k\right)_{\mathbb{R}^{m}}>0 \quad \forall k \in K \backslash\{0\} .
$$

Доведемо існування розв'язків екстремальної задачі

$$
\begin{aligned}
& \left(k^{*}, J(u)\right)_{\mathbb{R}^{m}} \rightarrow \inf _{u \in U} \\
& \mathcal{L} y=F(u), \quad u \in U .
\end{aligned}
$$

Розглянемо мінімізуючу послідовність $\left(u_{p}\right)$ допустимих керувань, тобто,

$$
u_{p} \in U, \quad \lim _{p \rightarrow \infty}\left(k^{*}, J\left(u_{p}\right)\right)_{\mathbb{R}^{m}}=\inf _{u \in U}\left(k^{*}, J(u)\right)_{\mathbb{R}^{m}} .
$$

Оскільки $U-$ слабко компактна множина, то можна вважати, що

$$
u_{p} \rightarrow u^{*} \in U \quad \text { слабко в } V .
$$

З урахуванням слабкої неперервності оператора $F: V \rightarrow H_{2}^{-}$маємо, що

$$
F\left(u_{p}\right) \rightarrow F\left(u^{*}\right) \text { слабко в } W_{2}^{-},
$$

зокрема, послідовність елементів $F\left(u_{p}\right)$ обмежена у просторі $W_{2}^{-}$.

Із теореми 1 випливає, що для довільного $p \in \mathbb{N}$ існує єдиний елемент $y_{p}=y\left(u_{p}\right) \in H_{1}$ такий, що $\left\langle y_{p}, \mathcal{L}^{+} p\right\rangle_{H_{1}, H_{1}^{-}}=\left\langle F\left(u_{p}\right), p\right\rangle_{W_{2}^{-}, W_{2}} \forall p \in W_{2}:$ $\mathcal{L}^{+} p \in H_{1}^{-}, \mathrm{i}\left\|y_{p}\right\|_{H_{1}} \leq c\left\|F\left(u_{p}\right)\right\|_{W_{2}^{-}}$. Послідовність $\left(y_{p}\right)$ обмежена у простоpi $H_{1}$, а отже, з неї можна виділити підпослідовність (яку знову позначимо $\left.\left(y_{p}\right)\right)$, слабко збіжну у просторі $H_{1}$ до елемента $y^{*} \in H_{1}$. Здійснивши у вищенаведеній тотожності граничний перехід при $p \rightarrow \infty$, одержимо

$$
\left\langle y^{*}, \mathcal{L}^{+} p\right\rangle_{H_{1}, H_{1}^{-}}=\left\langle F\left(u^{*}\right), p\right\rangle_{W_{2}^{-}, W_{2}} \quad \forall p \in W_{2}: \quad \mathcal{L}^{+} p \in H_{1}^{-},
$$


тобто, $y^{*} \in H_{1}-$ узагальнений розв'язок (1), що відповідає керуванню $u^{*} \in U .3$ огляду на слабку напівнеперервність знизу функціонала

$$
H_{1} \text { э } y \mapsto \frac{1}{2} \sum_{i=1}^{m} k_{i}^{*}\left\|C_{i} y-a_{i}\right\|_{E_{i}}^{2}
$$

маємо

$$
\left(k^{*}, J\left(u^{*}\right)\right)_{\mathbb{R}^{m}} \leq \liminf _{p \rightarrow \infty}\left(k^{*}, J\left(u_{p}\right)\right)_{\mathbb{R}^{m}}=\inf _{u \in U}\left(k^{*}, J(u)\right)_{\mathbb{R}^{m}} .
$$

Покажемо, що $u^{*} \in E_{K}(U)$. Від супротивного. Припустимо, що існує керування $v \in U$ таке, що $J(v)-J\left(u^{*}\right) \in-(K \backslash\{0\})$. Тоді згідно з вибором вектора $k^{*}$ маємо $\left(k^{*}, J(v)\right)_{\mathbb{R}^{m}}<\left(k^{*}, J\left(u^{*}\right)\right)_{\mathbb{R}^{m}}$. Отримане протиріччя доводить теорему.

Аналогічно доводиться

Теорема 3. Нехай виконано умови:

1) $U$ - компактна підмножина простору Гілъберта $V$;

2) $\mathrm{F}: \mathrm{V} \rightarrow \mathrm{H}_{2}^{-}$- неперервний оператор.

Тоді множина ефективних керувань $E_{K}(U)$ непорожня.

Зауваження 1. У загальному некомпактному випадку множини допустимих керувань $U \subseteq V$ множина ефективних розв'язків $E_{K}(U)$ може бути порожньою. Однак, відповідним чином послабивши поняття ефективного розв'язку, можна одержати деякі змістовні результати про існування розв'язків і в цій ситуації, що буде зроблено нижче.

Розглянемо апроксимацію задачі (5) сингулярного векторного оптимального керування скінченновимірною задачею.

Припустимо, що $V$ - сепарабельний простір Гільберта. Тоді існує послідовність лінійних скінченновимірних підпросторів $V_{s} \subseteq V$, яка задовольняє умову граничної щільності: $\lim _{s \rightarrow \infty} \inf _{u^{\prime} \in V_{s}}\left\|u^{\prime}-u\right\|_{V}=0(u \in V)$.

Нехай $U-$ слабко компактна підмножина простору керувань $V$. Розглянемо послідовність $\left(U_{s}\right)$ замкнених опуклих підмножин $V_{s}$, що рівномірно по $s$ обмежені в нормі простору $V$ та апроксимують множину допустимих керувань $U$ у наступному розумінні:

$$
\begin{gathered}
\forall u \in U \exists u_{s} \in U_{s}: u_{s} \underset{s \rightarrow \infty}{\longrightarrow} u \text { сильно в } V, \\
\forall u \in V, u_{s} \in U_{s}: u_{s} \underset{s \rightarrow \infty}{\longrightarrow} u \text { слабко в } V \Rightarrow u \in U .
\end{gathered}
$$

Задачу (5), тобто,

$$
\begin{gathered}
J(u) \rightarrow K-\min , \\
\mathcal{L} y=F(u), \quad u \in U
\end{gathered}
$$

апроксимуємо наступною задачею

$$
\begin{gathered}
J(u) \rightarrow K-\min , \\
\mathcal{L} y=F(u), u \in U_{s} \subseteq V_{s} \subseteq V .
\end{gathered}
$$

Має місце 
Твердження 1. Нехай $\mathbb{R}_{+}^{m} \cap \operatorname{int} K^{*} \neq \emptyset i$ відображення $F: V \rightarrow W_{2}^{-}$ слабко неперервне. Тоді для кожного $s \in \mathbb{N}$ множина $E_{K}\left(U_{s}\right)$ ефђективних розв'язків задачі (12), (13) непорожня.

Візьмемо довільний вектор $k^{*} \in \mathbb{R}_{+}^{m} \cap \operatorname{int} K^{*} \neq \emptyset$ та розглянемо екстремальні задачі

$$
\begin{gathered}
\left(k^{*}, J(u)\right)_{\mathbb{R}^{m}} \rightarrow \inf , \\
\mathcal{L} y=F(u), u \in U_{s} \subseteq V_{s} \subseteq V
\end{gathered}
$$

та

$$
\begin{aligned}
& \left(k^{*}, J(u)\right)_{\mathbb{R}^{m}} \rightarrow \inf , \\
& \mathcal{L} y=F(u), u \in U .
\end{aligned}
$$

Зрозуміло, що (14), (15) і (16), (17) мають розв'язки, причому всі вони належать множинам $E_{K}\left(U_{s}\right)$ і $E_{K}(U)$ відповідно.

Припустимо, що

оператор $F: V \rightarrow W_{2}^{-}$неперервний та слабко неперервний.

Нехай $\bar{u} \in U \cap E_{K}(U)$ - деяке оптимальне керування системою (16), (17), а $\left(\bar{u}_{s}\right): \bar{u}_{s} \in U_{s} \cap E_{K}\left(U_{s}\right)$ - послідовність розв'язків задач (14), (15). Тоді за умовою (8) існує послідовність $u_{s} \in U_{s}$ така, що $\left\|u_{s}-\bar{u}\right\|_{V} \rightarrow 0$ при $s \rightarrow \infty$.

Через сильну неперервність оператора $F: V \rightarrow W_{2}^{-}$маємо

$$
\left\|F\left(u_{s}\right)-F(\bar{u})\right\|_{W_{2}^{-}} \rightarrow 0 \text { при } s \rightarrow \infty .
$$

3 (19) та нерівності

$$
\left\|y_{s}-\bar{y}\right\|_{H_{1}} \leq C\left\|F\left(u_{s}\right)-F(\bar{u})\right\|_{W_{2}^{-}},
$$

де $y_{s}=y\left(u_{s}\right) \in H_{1}, \bar{y}=y(\bar{u}) \in H_{1}-$ узагальнені розв'язки рівняння стану системи (1), які відповідають керуванням $u_{s}, \bar{u}$, випливає, що $\left\|y_{s}-\bar{y}\right\|_{H_{1}} \rightarrow$ 0 при $s \rightarrow \infty$. Оскільки функціонал

$$
H_{1} \text { э } y \mapsto \frac{1}{2} \sum_{i=1}^{m} k_{i}^{*}\left\|C_{i} y-a_{i}\right\|_{E_{i}}^{2}
$$

є сильно неперервним, то

$$
\begin{aligned}
\limsup _{s \rightarrow \infty}\left(k^{*}, J\left(\bar{u}_{s}\right)\right)_{\mathbb{R}^{m}} \leq \lim _{s \rightarrow \infty}\left(k^{*}, J\left(u_{s}\right)\right)_{\mathbb{R}^{m}}= \\
\quad=\left(k^{*}, J(\bar{u})\right)_{\mathbb{R}^{m}}=\inf _{u \in U}\left(k^{*}, J(u)\right)_{\mathbb{R}^{m}} .
\end{aligned}
$$

Розглянемо послідовність $\left(\bar{u}_{s}\right)$. Множини $U_{s}$ рівномірно по $s$ обмежені у гільбертовому просторі $V$. Отже, існує підпослідовність $\left(\bar{u}_{s_{k}}\right)$ і елемент $u^{\prime} \in V$ такі, що $\bar{u}_{s_{k}} \rightarrow u^{\prime}$ слабко в $V$.

Із апроксимаційної умови (9) випливає, що $u^{\prime} \in U$. Оператор $F: V \rightarrow$ $W_{2}^{-}$- слабко неперервний (умова $(18)$ ), тому $F\left(\bar{u}_{s_{k}}\right) \rightarrow F\left(u^{\prime}\right)$ слабко в $W_{2}^{-}$. Послідовність $F\left(\bar{u}_{s_{k}}\right)$ обмежена в нормі простору $W_{2}^{-}$. Тому з оцінки

$$
\left\|y\left(\bar{u}_{s_{k}}\right)\right\|_{H_{1}} \leq C\left\|F\left(\bar{u}_{s_{k}}\right)\right\|_{W_{2}^{-}}
$$


маємо, що $\left(y\left(\bar{u}_{s_{k}}\right)\right)$ - обмежена в $H_{1}$ послідовність. Виділяємо з послідовності $\left(y\left(\bar{u}_{s_{k}}\right)\right)$ слабко збіжну підпослідовність $\left(y\left(\bar{u}_{s_{k_{l}}}\right)\right)$ :

$$
y\left(\bar{u}_{s_{k_{l}}}\right) \rightarrow y^{\prime} \text { слабко в } H_{1} .
$$

Покажемо, що $y^{\prime}=y\left(u^{\prime}\right)$. Справді, маємо

$$
\left\langle y\left(\bar{u}_{s_{k_{l}}}\right), \mathcal{L}^{+} p\right\rangle_{H_{1}, H_{1}^{-}}=\left\langle F\left(\bar{u}_{s_{k_{l}}}\right), p\right\rangle_{W_{2}^{-}, W_{2}} \quad \forall p \in W_{2}: \quad \mathcal{L}^{+} p \in H_{1}^{-},
$$

Здійснивши граничний перехід в (22) при $l \rightarrow \infty$, отримаємо

$$
\left\langle y^{\prime}, \mathcal{L}^{+} p\right\rangle_{H_{1}, H_{1}^{-}}=\left\langle F\left(u^{\prime}\right), p\right\rangle_{W_{2}^{-}, W_{2}} \quad \forall p \in W_{2}: \quad \mathcal{L}^{+} p \in H_{1}^{-} .
$$

Отже, $y^{\prime}=y\left(u^{\prime}\right) \in H_{1}-$ узагальнений розв'язок рівняння стану системи (1), що відповідає керуванню $u^{\prime} \in U$. Оскільки цей розв'язок єдиний, то і вся послідовність $\left(y\left(\bar{u}_{s_{k}}\right)\right)$ слабко в $H_{1}$ збігається до $y\left(u^{\prime}\right) \in H_{1}$.

Покажемо, що елемент $u^{\prime} \in U-$ розв'язок задачі (16), (17). Із слабкої напівнеперервності знизу функціонала (20) та нерівності (21) випливає, що

$$
\begin{aligned}
\left(k^{*}, J\left(u^{\prime}\right)\right)_{\mathbb{R}^{m}} \leq \liminf _{s \rightarrow \infty}\left(k^{*}, J\left(\bar{u}_{s_{k}}\right)\right)_{\mathbb{R}^{m}} \leq \\
\quad \leq \limsup _{s \rightarrow \infty}\left(k^{*}, J\left(\bar{u}_{s_{k}}\right)\right)_{\mathbb{R}^{m}} \leq \inf _{u \in U}\left(k^{*}, J(u)\right)_{\mathbb{R}^{m}} .
\end{aligned}
$$

Отже, $\left(k^{*}, J\left(u^{\prime}\right)\right)_{\mathbb{R}^{m}}=\inf _{u \in U}\left(k^{*}, J(u)\right)_{\mathbb{R}^{m}}$ i $u^{\prime} \in U-$ розв'язок задачі

$$
\begin{aligned}
& \left(k^{*}, J(u)\right)_{\mathbb{R}^{m}} \rightarrow \inf , \\
& \mathcal{L} y=F(u), u \in U .
\end{aligned}
$$

Підсумовуючи, можемо сформулювати теорему.

Теорема 4. Нехай $\mathbb{R}_{+}^{m} \cap \operatorname{int} K^{*} \neq \emptyset$, множина $U \subseteq V$ слабко компактна, виконуютвся умови (8), (9) та (18). Тоді для кожного $k^{*} \in \mathbb{R}_{+}^{m} \cap \operatorname{int} K^{*}$ задача оптимального керування

$$
\begin{gathered}
\left(k^{*}, J(u)\right)_{\mathbb{R}^{m}} \rightarrow \inf , \\
\mathcal{L} y=F(u), u \in U_{s} \subseteq V_{s} \subseteq V
\end{gathered}
$$

мае розв'язок $\bar{u}_{s} \in U_{s} \cap E_{K}\left(U_{s}\right)$ та усі слабкі граничні точки послідовності $\left(\bar{u}_{s}\right)$ належать множині $E_{K}(U)$ розв'язків задачі

$$
J(u) \rightarrow K-\min , \quad \mathcal{L} y=F(u), u \in U .
$$

\section{3. УМови ЕФЕКТИВНостІ}

Розглянемо систему, що описується рівнянням

$$
\mathcal{L} y=F(u), u \in U
$$

3 векторним критерієм якості

$$
J(u)=\left(\begin{array}{c}
\frac{1}{2}\left\|C_{1} y(u)-a_{1}\right\|_{E_{1}}^{2} \\
\cdots \\
\frac{1}{2}\left\|C_{m} y(u)-a_{m}\right\|_{E_{m}}^{2}
\end{array}\right) .
$$

У теоремах 5 і 6 дамо відповідь на питання про вигляд та властивості похідної критерію якості. 
Теорема 5. Нехай відображення $F: V \rightarrow W_{2}^{-}$диференційовне у точці $u \in$ $V$ за Гато (Фреше) $і F^{\prime}(u) \in L\left(V, W_{2}^{-}\right)$- відповідна похідна. Тоді існуе похідна Гато (Фреше) $J^{\prime}(u)$ відображення $J: V \rightarrow \mathbb{R}^{m}$, яка визначається формулою

$$
J^{\prime}(u)=\left(\begin{array}{c}
\left(F^{\prime}(u)\right)^{*} p_{1} \\
\cdots \\
\left(F^{\prime}(u)\right)^{*} p_{m}
\end{array}\right)
$$

де $p_{i} \in W_{2}-$ розв'язок операторного рівняння

$$
\mathcal{L}^{+} p_{i}=C_{i}^{*} C_{i} y(u)-C_{i}^{*} a_{i}, \quad i=\overline{1, m} .
$$

Теорема 6. Справджуютвся твердження:

1) якщо у деякій точиі $\bar{u} \in V$ відображення $u \mapsto F^{\prime}(u)$ неперервне, то $i$ відображення $u \mapsto J^{\prime}(u)$ неперервне в $\bar{u} \in V$;

2) якщо на обмеженій опуклій множсин $O \subseteq V$ відображення $u \mapsto$ $F^{\prime}(u)$ задовольняе умову Гельдера з показником $\gamma \in(0,1]$, то $i$ відображення $u \mapsto J^{\prime}(u)$ задовольняе на множині $O$ умову Гельдера з показником $\gamma$.

Далі будемо вважати, що відображення $F: V \rightarrow W_{2}$ диференційовне за Фреше у точках допустимої множини $U$.

Перейдемо до умов локальної ефективності керувань в задачах (5)-(7).

Нагадаємо класичну конструкцію конуса дотичних напрямків [22].

Замкнений конус

$$
\begin{aligned}
& T\left(u_{0} \mid U\right)=\limsup _{\alpha \rightarrow+0} \frac{U-u_{0}}{\alpha}= \\
& =\left\{v \in V: \exists\left(\alpha_{k}>0, v_{k} \in U\right), \quad \alpha_{k} \rightarrow+0, \frac{v_{k}-u_{0}}{\alpha_{k}} \rightarrow v \text { при } k \rightarrow \infty\right\}
\end{aligned}
$$

називається дотичним або контингентним до непорожньої множини $U$ в точці $u_{0} \in \mathrm{cl} U$.

Відмітимо, що $T\left(u_{0} \mid U\right)=\{0\}$ тільки тоді, коли $u_{0}$ - ізольована точка множини $U$. Очевидно, що в цьому випадку точка $u_{0}$ - локально ефективна. Отже, будемо завжди припускати, що $T\left(u_{0} \mid U\right) \neq\{0\}$.

Зауваження 2. Існує декілька конструкцій дотичних конусів (див. огляд у [22]). Наведену тут запропоновано у 30-х pp. ХХ ст. Ж. Буліганом. У теорії оптимізації подібна конструкція вперше з'явилась, можливо, в [23].

Розглянемо задачу пошуку слабко ефективних керувань

$$
\begin{aligned}
& J(u) \rightarrow K-\mathrm{w}-\min , \\
& \mathcal{L} y=F(u), u \in U .
\end{aligned}
$$

Має місце

Теорема 7. Нехай $\bar{u} \in \operatorname{loc} W E_{K}(U)$. Tодi

$$
\forall w \in T(\bar{u} \mid U) \backslash\{0\}:\left(\begin{array}{c}
\left(\left(F^{\prime}(\bar{u})\right)^{*} p_{1}, w\right)_{V} \\
\cdots \\
\left(\left(F^{\prime}(\bar{u})\right)^{*} p_{m}, w\right)_{V}
\end{array}\right) \notin-\operatorname{int} K,
$$


de $p_{i} \in W_{2}: \mathcal{L}^{+} p_{i}=C_{i}^{*} C_{i} y(\bar{u})-C_{i}^{*} a_{i}, i=\overline{1, m}$.

Доведення. Нехай $\bar{u} \in \operatorname{loc} W E_{K}(U)$ і $w \in T(\bar{u} \mid U) \backslash\{0\}$. Тоді за означенням множини $\operatorname{loc} W E_{K}(U)$ та конуса $T(\bar{u} \mid U)$ маємо

$$
J(v)-J(\bar{u}) \notin-\operatorname{int} K \quad \forall v \in U \cap O(\bar{u})
$$

та

$$
J\left(v_{k}\right)-J(\bar{u})=J\left(\bar{u}+v_{k}-\bar{u}\right)-J(\bar{u}) \notin-\operatorname{int} K \quad \forall k \geq k_{0} .
$$

Далі,

$$
J^{\prime}(\bar{u})\left(\frac{v_{k}-\bar{u}}{\alpha_{k}}\right)+o(1)\left\|\frac{v_{k}-\bar{u}}{\alpha_{k}}\right\|_{V} \notin-\operatorname{int} K .
$$

Зробивши в (24) граничний перехід при $k \rightarrow \infty$ з урахуванням замкненості множини $\mathbb{R}^{m} \backslash(-\operatorname{int} K)$, одержимо включення $J^{\prime}(\bar{u})(w) \notin-\operatorname{int} K$, яке можна записати у вигляді (23).

Якщо ввести конус $S D_{J}(u)=\left\{w \in V: J^{\prime}(u)(w) \in-\operatorname{int} K\right\}$ напрямків спадання відображення $J: V \rightarrow \mathbb{R}^{m}$ у точці $u \in V$, то включення (23) рівносильне співвідношенню

$$
S D_{J}(\bar{u}) \cap T(\bar{u} \mid U)=\emptyset .
$$

У випадку опуклості множини допустимих керувань співвідношення (23) можна подати у більш простій формі.

Теорема 8. Нехай $U-$ опукла підмножина $V i \bar{u} \in \operatorname{loc} W E_{K}(U)$. Тодi

$$
\forall v \in U:\left(\begin{array}{c}
\left(\left(F^{\prime}(\bar{u})\right)^{*} p_{1}, v-\bar{u}\right)_{V} \\
\cdots \\
\left(\left(F^{\prime}(\bar{u})\right)^{*} p_{m}, v-\bar{u}\right)_{V}
\end{array}\right) \notin-\operatorname{int} K,
$$

de $p_{i} \in W_{2}: \mathcal{L}^{+} p_{i}=C_{i}^{*} C_{i} y(\bar{u})-C_{i}^{*} a_{i}, i=\overline{1, m}$.

Доведення. Покажемо, що $\forall v \in U$ вектор $v-\bar{u}$ належить конусу $T(\bar{u} \mid U)$. Розглянемо послідовності $\beta_{k} \in(0,1), \beta_{k} \rightarrow 1$ i $v_{k}=\left(1-\beta_{k}\right) v+\beta_{k} \bar{u} \in U$. Звідси $v_{k}-\bar{u}=\left(1-\beta_{k}\right)(v-\bar{u})$. Отже, $v-\bar{u} \in T(\bar{u} \mid U)$, а тоді (23) тягне за собою виконання $(26)$.

Очевидно, що і зворотна імплікація $(26) \Rightarrow(23)$ справедлива у випадку опуклості множини $U$.

Отримаємо достатню умову локальної оптимальності для задачі

$$
\begin{gathered}
J(u) \rightarrow K \text {-s- min, } \\
\mathcal{L} y=F(u), u \in U .
\end{gathered}
$$

Має місце

Теорема 9. Нехай простір $V$ скінченновимірний, $\bar{u} \in U$ i виконано умову:

$$
\forall w \in T(\bar{u} \mid U) \backslash\{0\}:\left(\begin{array}{c}
\left(\left(F^{\prime}(\bar{u})\right)^{*} p_{1}, w\right)_{V} \\
\cdots \\
\left(\left(F^{\prime}(\bar{u})\right)^{*} p_{m}, w\right)_{V}
\end{array}\right) \notin-K,
$$

де $p_{i} \in W_{2}: \mathcal{L}^{+} p_{i}=C_{i}^{*} C_{i} y(\bar{u})-C_{i}^{*} a_{i}, i=\overline{1, m}$. Tодi $\bar{u} \in \operatorname{loc} S E_{K}(U)$. 
Доведення. Припустимо, що має місце умова $(27)$, однак $\bar{u} \notin \operatorname{loc} S E_{K}(U)$. Тоді існує послідовність $v_{k} \in U \backslash\{u\}$ така, що $v_{k} \rightarrow \bar{u}, \frac{v_{k}-\bar{u}}{\left\|v_{k}-\bar{u}\right\|_{V}} \rightarrow w \in$ $T(\bar{u} \mid U) \backslash\{0\}$ і $J\left(v_{k}\right)-J(\bar{u}) \in-K$. Далі маємо

$$
\frac{J\left(v_{k}\right)-J(\bar{u})}{\left\|v_{k}-\bar{u}\right\|_{V}}=J^{\prime}(\bar{u})\left(\frac{v_{k}-\bar{u}}{\left\|v_{k}-\bar{u}\right\|_{V}}\right)+o(1) \in-K .
$$

Зробивши в (28) граничний перехід при $k \rightarrow \infty$, одержимо $J^{\prime}(\bar{u})(w) \in-K$, що суперечить (27).

Якщо ввести конус $D_{J}(u)=\left\{w \in V: J^{\prime}(u)(w) \in-K\right\}$ напрямків нестрогого спадання відображення $J: V \rightarrow \mathbb{R}^{m}$ у точці $u \in V$, то включення (27) рівносильне подібному (25) співвідношенню

$$
D_{J}(\bar{u}) \cap T(\bar{u} \mid U)=\{0\} .
$$

Розглянемо варіант скаляризації необхідних умов ефективності. Відповідні скалярні умови використаємо для побудови ітераційних алгоритмів розв'язання задач керування з векторним критерієм якості.

Розглянемо компакт $B \subseteq \mathbb{R}^{m}$ такий, що не містить нуль і $K^{*}=\operatorname{con}(\operatorname{conv} B)$. Якщо $K=K^{*}=\mathbb{R}_{+}^{m}$, то можна покласти $B=\left\{e_{1}, e_{2}, \ldots, e_{m}\right\}$, де

$$
e_{i}=(0, \ldots, 0, \underset{i \text {-та позиція }}{1}, 0, \ldots, 0) .
$$

У загальному випадку покладемо, наприклад,

$$
B=K^{*} \cap\left\{k \in \mathbb{R}^{m}:\|k\|_{\mathbb{R}^{m}}=1\right\} .
$$

Конуси $K$ та $\operatorname{int} K$ можна подати у вигляді

$$
\begin{gathered}
K=\left\{x \in \mathbb{R}^{m}:\left(x, x^{\prime}\right)_{\mathbb{R}^{m}} \geq 0 \forall x^{\prime} \in B\right\}, \\
\operatorname{int} K=\left\{x \in \mathbb{R}^{m}:\left(x, x^{\prime}\right)_{\mathbb{R}^{m}}>0 \forall x^{\prime} \in B\right\} .
\end{gathered}
$$

Розглянемо опорну функцію множини $B$

$$
\sigma_{B}(x)=\max _{x^{\prime} \in B}\left(x, x^{\prime}\right)_{\mathbb{R}^{m}} \quad \forall x \in \mathbb{R}^{m} .
$$

Безпосередньо з (29) випливає додатня однорідність, напівадитивність та ліпшицевість функції $\sigma_{B}$. Із компактності та породжуючої властивості множини $B$ випливають потрібні нам представлення

$$
\begin{gathered}
-K=\left\{x \in \mathbb{R}^{m}: \sigma_{B}(x) \leq 0\right\}, \\
-\operatorname{int} K=\left\{x \in \mathbb{R}^{m}: \sigma_{B}(x)<0\right\} .
\end{gathered}
$$

Ці представлення конусів $-K$ та $-\operatorname{int} K$ дозволяють нам переформулювати умови ефективності так.

Наслідок 1. Нехай $\bar{u} \in \operatorname{loc} W E_{K}(U)$. Тодi

$$
\begin{gathered}
\forall w \in T(\bar{u} \mid U) \backslash\{0\}: \sigma_{B} \circ\left(\left(F^{\prime}(\bar{u})\right)^{*} \vec{p}\right)(w) \geq 0, \\
\text { de } \vec{p}=\left(p_{1}, p_{2}, \ldots, p_{m}\right) \in\left(W_{2}\right)^{m}: \mathcal{L}^{+} p_{i}=C_{i}^{*} C_{i} y(\bar{u})-C_{i}^{*} a_{i}, i=\overline{1, m} .
\end{gathered}
$$


Наслідок 2. Нехай $U-$ опукла підмножина $V i \bar{u} \in \operatorname{loc} W E_{K}(U)$. Тодi

$$
\forall v \in U: \sigma_{B} \circ\left(\left(F^{\prime}(\bar{u})\right)^{*} \vec{p}\right)(v-\bar{u}) \geq 0,
$$

de $\vec{p}=\left(p_{1}, p_{2}, \ldots, p_{m}\right) \in\left(W_{2}\right)^{m}: \mathcal{L}^{+} p_{i}=C_{i}^{*} C_{i} y(\bar{u})-C_{i}^{*} a_{i}, i=\overline{1, m}$.

Наслідок 3. Нехай простір $V$ скінченновимірний, $\bar{u} \in U$ i виконано умову:

$$
\forall w \in T(\bar{u} \mid U) \backslash\{0\}: \sigma_{B} \circ\left(\left(F^{\prime}(\bar{u})\right)^{*} \vec{p}\right)(w)>0,
$$

де $\vec{p}=\left(p_{1}, p_{2}, \ldots, p_{m}\right) \in\left(W_{2}\right)^{m}: \mathcal{L}^{+} p_{i}=C_{i}^{*} C_{i} y(\bar{u})-C_{i}^{*} a_{i}, i=\overline{1, m}$. Тодi $\bar{u} \in \operatorname{loc} S E_{K}(U)$.

Відмітимо, якщо виконано $x-y \in K(x-y \in \operatorname{int} K)$, то $\sigma_{B}(x) \geq \sigma_{B}(y)$ $\left(\sigma_{B}(x)>\sigma_{B}(y)\right)$. Дійсно, маємо $\sigma_{B}(y-x) \leq 0\left(\sigma_{B}(y-x)<0\right)$. Але

$$
\sigma_{B}(x)+\sigma_{B}(y-x) \geq \sigma_{B}(y),
$$

звідки випливає бажане.

Зауваження 3. Розглянемо деяку множину $X$ і відображення

$$
X \stackrel{J}{\longrightarrow} \mathbb{R}^{m} .
$$

Задачу пошуку точок $\bar{x} \in X$ таких, що $J(X) \cap(J(\bar{x})-\operatorname{int} K)=\emptyset$, можна переформулювати у вигляді:

$$
\text { знайти } \bar{x} \in X: g(\bar{x}, x) \geq 0 \forall x \in X \text {, }
$$

де $g\left(x_{1}, x_{2}\right)=\sigma_{B}\left(J\left(x_{2}\right)-J\left(x_{1}\right)\right)$. Задача (33) суть загальна задача рівноважного програмування. Отже, популярна останнім часом «рівноважна» алгоритміка $[24,25]$ може бути використана для побудови методів розв'язання розглянутих у цьому розділі задач.

\section{4. АПРОКСИМАЦІЙНА ЕФЕКТИВНІСТЬ}

Розглянемо докладно ослаблення поняття ефективного розв'язку, про яке згадували у зауваженні 1.

Оберемо та зафіксуємо довільний елемент $e \in \operatorname{int} K$. Має місце

Твердження 2. Якщо відображення $g: U \rightarrow \mathbb{R}^{m} K$-обмежене знизу (тобто, $\left.\exists k \in \mathbb{R}^{m}: g(U) \subseteq k+K\right)$, то для довільного $\varepsilon>0$ існуе такий елемент $u_{\varepsilon} \in U$, що

$$
\forall v \in U: g(v) \notin g\left(u_{\varepsilon}\right)-\varepsilon \cdot e-(K \backslash\{0\}) .
$$

Доведення. Візьмемо довільний вектор $k^{*} \in K^{*} \backslash\{0\}$. Тоді для всіх $v \in U$ маємо

$$
\left(k^{*}, g(v)\right)_{\mathbb{R}^{m}} \geq\left(k^{*}, k\right)_{\mathbb{R}^{m}} .
$$

Покладемо $s=\inf _{v \in U}\left(k^{*}, g(v)\right)_{\mathbb{R}^{m}}$. За умовою $s>-\infty \mathrm{i}\left(k^{*}, e\right)_{\mathbb{R}^{m}}>0$. Очевидно, що для кожного $\varepsilon>0$ існує елемент $u_{\varepsilon} \in U$ такий, що

$$
s \leq\left(k^{*}, g\left(u_{\varepsilon}\right)\right)_{\mathbb{R}^{m}}<s+\varepsilon\left(k^{*}, e\right)_{\mathbb{R}^{m}} \leq\left(k^{*}, g(v)\right)_{\mathbb{R}^{m}}+\varepsilon\left(k^{*}, e\right)_{\mathbb{R}^{m}} \quad \forall v \in U .
$$

Припустимо, що існує $u^{\prime} \in U$ такий, що $g\left(u^{\prime}\right) \in g\left(u_{\varepsilon}\right)-\varepsilon \cdot e-(K \backslash\{0\})$. Тоді

$$
\left(k^{*}, g\left(u^{\prime}\right)\right)_{\mathbb{R}^{m}}+\varepsilon \cdot\left(k^{*}, e\right)_{\mathbb{R}^{m}} \leq\left(k^{*}, g\left(u_{\varepsilon}\right)\right)_{\mathbb{R}^{m}} .
$$


Отримане протиріччя доводить твердження.

Зауваження 4. Оскільки $\operatorname{int} K \neq \emptyset$, то з обмеженості множини $g(U)$ випливає $K$-обмеженість знизу відображення $g: U \rightarrow \mathbb{R}^{m}$.

Розглянемо задачу керування

$$
\begin{gathered}
J(u) \rightarrow K \text { - min, } \\
\mathcal{L} y=F(u), \quad u \in U .
\end{gathered}
$$

Із зауваження 4 випливає, що для $K$-обмеженості знизу відображення $J(\cdot)$ достатньо обмеженості множини допустимих керувань $U \subseteq V$ та неперервності оператора $F: V \rightarrow W_{2}^{-}$.

Твердження 2 дозволяе природно ввести наступне ослаблення поняття ефективного розв'язку задачі (34), (35). Нехай $\varepsilon>0$ i $e \in \operatorname{int} K$.

Означення 1. Елемент $u_{\varepsilon} \in U$ називаємо $(K, e, \varepsilon)$-апроксимаційно ефективним розв'язком задачі (34), (35), якщо

$$
\forall v \in U: \quad J(v) \notin J\left(u_{\varepsilon}\right)-\varepsilon \cdot e-(K \backslash\{0\}) .
$$

Множину усіх $(K, e, \varepsilon)$-апроксимаційно ефективних розв'язків задачі (34), (35) позначимо символом $E_{(K, e, \varepsilon)}(U)$.

$\mathrm{У}$ скалярному випадку $(K, e, \varepsilon)$-апроксимаційно ефективний розв'язок це просто $\varepsilon$-оптимальний (субоптимальний) розв'язок. У наведеному вигляді поняття $(K, e, \varepsilon)$-апроксимаційно ефективного розв'язку задачі багатокритеріальної оптимізації вперше запропоновано та систематично застосовувалося для дослідження багатокритеріальних задач розміщення об'єктів та теорії наближення $\mathrm{K}$. Таммер [26]. Введення $(K, e, \varepsilon)$-апроксимаційно ефективних розв'язків виправдане тим, що множини ефективних розв'язків, як правило, порожні у загальному некомпактному випадку, у той час як апроксимаційно ефективні розв'язки існують при досить слабких припущеннях. Крім того ясно, що чисельні методи після виконання скінченного числа ітерацій, як правило, генерують тільки наближені розв'язки.

Для отримання необхідних умов першого порядку $(K, e, \varepsilon)$-апроксимаційної ефективності використаємо аналог класичного варіаційного принципу Екланда для векторнозначних відображень.

Сформулюємо далеко не найзагальніший, але достатній для наших застосувань аналог теореми Екланда для векторнозначних відображень [27]. Нехай $E, F$ - банахові простори, простір $F$ частково упорядковано гострим замкненим опуклим конусом $K \subseteq F$ з непорожньою внутрішністю, $e \in \operatorname{int} K$.

Теорема 10. Нехай виконано умови:

1) $X$ - замкнена підмножина банахова простору $E$;

2) відображсення $f: X \rightarrow F K$-обмежене знизу $i K$-напівнеперервне знизу.

Тоді для довільних $\varepsilon>0, \delta>0$ i точки $x_{0} \in U$

$$
f(X) \cap\left(f\left(x_{0}\right)-\varepsilon \cdot e-(K \backslash\{0\})\right)=\emptyset
$$

існуе така точка $x_{\varepsilon} \in X$, що: 
1) $f\left(x_{\varepsilon}\right) \in f\left(x_{0}\right)-\varepsilon \delta^{-1}\left\|x_{0}-x_{\varepsilon}\right\|_{E} \cdot e-(K \backslash\{0\})$;

2) $\left\|x_{0}-x_{\varepsilon}\right\|_{E} \leq \delta$

3) $\forall x \in X: f(x)+\varepsilon \delta^{-1}\left\|x-x_{\varepsilon}\right\|_{E} e \notin f\left(x_{\varepsilon}\right)-(K \backslash\{0\})$, mобто $x_{\varepsilon} \in X$ - ефективна точка відображення $x \mapsto f(x)+\varepsilon \delta^{-1}\left\|x-x_{\varepsilon}\right\|_{E}$ e на множині $X$.

Теорема 10 стверджує існування ефективного розв'язку збуреної задачі $f(x)+\frac{\varepsilon}{\delta}\left\|x-x_{\varepsilon}\right\|_{E} e \rightarrow K$ - min, $x \in X$ в $\delta$-околі $(K, e, \varepsilon)$-апроксимаційно ефективного розв'язку вихідної задачі $f(x) \rightarrow K$ - $\min , x \in X$, який у свою чергу завжди існуе для $K$-обмеженого знизу відображення $f: X \rightarrow F$. Висновок 3 теореми $10 є$ найважливішим та дозволяє одержати необхідні умови для $(K, e, \varepsilon)$-апроксимаційно ефективних розв'язків у вигляді варіаційних нерівностей.

Повернемось до задачі керування (34), (35). Має місце

Теорема 11. Нехай виконано умови:

1) $U-$ замкнена обмежена підмножина простору $V$;

2) $F: V \rightarrow W_{2}^{-}-$диференційовний за Фреше оператор.

Тоді для довільних $\varepsilon>0, \delta>0$ існують такі точки $\bar{u} \in U, \tilde{u} \in U$, що:

1) $\bar{u} \in E_{(K, e, \varepsilon)}(U)$;

2) $J(\tilde{u}) \in J(\bar{u})-(K \backslash\{0\})$;

3) $\|\bar{u}-\tilde{u}\|_{V} \leq \delta$

4) виконуеться

$\left(\begin{array}{c}\left(\left(F^{\prime}(\tilde{u})\right)^{*} p_{1}, w\right)_{V} \\ \cdots \\ \left(\left(F^{\prime}(\tilde{u})\right)^{*} p_{m}, w\right)_{V}\end{array}\right)+\frac{\varepsilon}{\delta}\|w\|_{V} e \notin-\operatorname{int} K \quad \forall w \in T(\tilde{u} \mid U) \backslash\{0\}$,

де $p_{i} \in W_{2}-$ розв'язок рівняння $\mathcal{L}^{+} p_{i}=C_{i}^{*} C_{i} y(\tilde{u})-C_{i}^{*} a_{i}, i=\overline{1, m}$.

Доведення. Завдяки умовам теореми ми можемо використовувати твердження 2 і теорему 10, тобто, для довільних $\varepsilon>0, \delta>0$ існують точки $\bar{u} \in U, \tilde{u} \in U$ такі, що:

$$
\begin{gathered}
\bar{u} \in E_{(K, e, \varepsilon)}(U) ; \quad J(\tilde{u}) \in J(\bar{u})-(K \backslash\{0\}) ;\|\bar{u}-\tilde{u}\|_{V} \leq \delta ; \\
\forall u \in U: J(u)-J(\tilde{u})+\varepsilon \delta^{-1}\|u-\tilde{u}\|_{V} e \notin-(K \backslash\{0\}) .
\end{gathered}
$$

Розглянемо довільний вектор $w \in T(\tilde{u} \mid U) \backslash\{0\}$. Тоді за означенням дотичного конуса $T(\tilde{u} \mid U)$ маємо $J\left(v_{k}\right)-J(\tilde{u})+\varepsilon \delta^{-1}\left\|v_{k}-\tilde{u}\right\|_{V} e \notin-(K \backslash\{0\})$ $\forall k \in \mathbb{N}$. Далі

$$
J^{\prime}(\tilde{u})\left(\frac{v_{k}-\tilde{u}}{\alpha_{k}}\right)+o(1)\left\|\frac{v_{k}-\tilde{u}}{\alpha_{k}}\right\|_{V}+\varepsilon \delta^{-1}\left\|\frac{v_{k}-\tilde{u}}{\alpha_{k}}\right\|_{V} e \notin-(K \backslash\{0\}) .
$$

Зробивши в (36) граничний перехід при $k \rightarrow \infty$ з урахуванням замкненості множини $\mathbb{R}^{m} \backslash(-\operatorname{int} K)$, одержимо $J^{\prime}(\bar{u})(w)+\varepsilon \delta^{-1}\|w\|_{V} e \notin-\operatorname{int} K$. Тобто, справджується співвідношення 4.

Якщо множина допустимих керувань $U \in$ замкненою опуклою підмножиною простору $V$, то умови $(K, e, \varepsilon)$-апроксимаційної ефективності приймають більш простий вигляд. 
Теорема 12. Нехай виконано умови теореми 11 i додатково

$$
U-\text { замкнена опукла підмножина простору } V \text {. }
$$

Тоді для довільних $\varepsilon>0, \delta>0$ існують такі точки $\bar{u} \in U, \tilde{u} \in U$, що:

1) $\bar{u} \in E_{(K, e, \varepsilon)}(U)$;

2) $J(\tilde{u}) \in J(\bar{u})-(K \backslash\{0\})$;

3) $\|\bar{u}-\tilde{u}\|_{V} \leq \delta$

4) виконуеться

$$
\left(\begin{array}{c}
\left(\left(F^{\prime}(\tilde{u})\right)^{*} p_{1}, v-\tilde{u}\right)_{V} \\
\cdots \\
\left(\left(F^{\prime}(\tilde{u})\right)^{*} p_{m}, v-\tilde{u}\right)_{V}
\end{array}\right)+\frac{\varepsilon}{\delta}\|v-\tilde{u}\|_{V} e \notin-\operatorname{int} K \quad \forall v \in U,
$$

де $p_{i} \in W_{2}-$ розв'язок рівняння $\mathcal{L}^{+} p_{i}=C_{i}^{*} C_{i} y(\tilde{u})-C_{i}^{*} a_{i}, i=\overline{1, m}$.

Зауваження 5. Якщо у теоремах 11, 12 покласти $\varepsilon=0$, то одержимо співвідношення теорем 7 і 8. Отримані у теоремі 12 умови $(K, e, \varepsilon)$ апроксимаційної ефективності можна використати при розробці і дослідженні чисельних методів оптимального керування.

\section{5. ЗБІЖНІСТЬ МОДЕЛЕЙ АЛГОРИТМІВ УЗАГАЛЬНЕНОЇ ОПТИМІЗАЦІЇ ЛІНІЙНИХ СИСТЕМ 3 ВЕКТОРНИМ КРИТЕРІЕМ ЯКОСТІ}

Розглянемо трохи загальнішу задачу. Нехай рівняння стану керованої системи має вигляд $\mathcal{L} y=F(u), u \in U$. Оператори $\mathcal{L}$ і $F$ задовольняють стандартні умови. Припустимо, що критерій якості має вигляд $J(u)=$ $\Phi(y(u), u)$, де $\Phi: H_{1} \times V \rightarrow \mathbb{R}^{m}, y(u)$ - стан системи, що відповідає керуванню $u \in U$.

Далі запропоновано та досліджено три методи градієнтного типу для розв'язання задачі

$$
\begin{gathered}
J(u)=\Phi(y(u), u) \rightarrow K-\min , \\
\mathcal{L} y(u)=F(u), \quad u \in U .
\end{gathered}
$$

Мають місце такі теореми - аналоги теорем 5 і 6.

Теорема 13. Нехай

1) оператор $F: V \rightarrow W_{2}^{-}$мае в точиі $u \in V$ похідну Фреше $F^{\prime}(u) \in$ $L\left(V, W_{2}^{-}\right)$

2) оператор $\Phi=\left(\Phi^{(1)}, \ldots, \Phi^{(m)}\right): H_{1} \times V \rightarrow \mathbb{R}^{m}$ має в точиі $(y(u), u) \in$ $H_{1} \times V$ похідну Фреше $i$ відповідні частинні похідні мають вигляд

$$
\begin{gathered}
\Phi_{1}^{\prime}(y(u), u)=\left(D_{1} \Phi^{(1)}(y(u), u), \ldots, D_{1} \Phi^{(m)}(y(u), u)\right) \in\left(H_{1}^{-}\right)^{m}, \\
\Phi_{2}^{\prime}(y(u), u)=\left(D_{2} \Phi^{(1)}(y(u), u), \ldots, D_{2} \Phi^{(m)}(y(u), u)\right) \in V^{m} .
\end{gathered}
$$


Тоді відображення $J=\left(J^{(1)}, \ldots, J^{(m)}\right): V \rightarrow \mathbb{R}^{m}$ диференційовне за Фреше в точиі $u \in V$ i його похідна обчислюетвся за формулоюо

$$
\begin{gathered}
J^{\prime}(u)(h)=\left(\begin{array}{c}
\left(D J^{(1)}(u), h\right)_{V} \\
\cdots \\
\left(D J^{(m)}(u), h\right)_{V}
\end{array}\right)= \\
\quad=\left(\begin{array}{c}
\left(\left(F^{\prime}(u)\right)^{*} p_{1}+D_{2} \Phi^{(1)}(y(u), u), h\right)_{V} \\
\cdots \\
\left(\left(F^{\prime}(u)\right)^{*} p_{m}+D_{2} \Phi^{(m)}(y(u), u), h\right)_{V}
\end{array}\right) \quad \forall h \in V,
\end{gathered}
$$

де $p_{i} \in W_{2}-$ розв'язок операторного рівняння $\mathcal{L}^{+} p_{i}=D_{1} \Phi^{(i)}(y(u), u)$, $i=\overline{1, m}$.

Теорема 14. Нехай на обмеженій опуклій множині $U \subseteq V$ оператор $u \mapsto F^{\prime}(u)$ задовольняе умову Гельдера з показником $\gamma \in(0,1]$, оператори $(y, u) \mapsto \Phi_{1}^{\prime}(y, u) i(y, u) \mapsto \Phi_{2}^{\prime}(y, u)$ задовольняють на обмежених підмножинах простору $H_{1} \times V$ умову Гельдера з показником $\gamma \in(0,1]$. Тодi похідна Фреше $J^{\prime}(\cdot)$ задовольняе на $U$ умову Гельдера з показником $\gamma$.

Далі будемо вважати, що множина допустимих керувань $U$ опукла, компактна і на ній виконуються умови теорем 13, 14.

Позначимо через $U^{*}$ множину керувань $u \in U$ таких, що

$$
\begin{gathered}
\left(\begin{array}{c}
\left(\left(F^{\prime}(u)\right)^{*} p_{1}+D_{2} \Phi^{(1)}(y, u), v-u\right)_{V} \\
\cdots \\
\left(\left(F^{\prime}(u)\right)^{*} p_{m}+D_{2} \Phi^{(m)}(y, u), v-u\right)_{V}
\end{array}\right) \notin-\operatorname{int} K \quad \forall v \in U, \\
\mathcal{L} y=F(u), \quad \mathcal{L}^{+} p_{i}=D_{1} \Phi^{(i)}(y, u), \quad i=\overline{1, m} .
\end{gathered}
$$

Тобто, $U^{*}-$ множина допустимих керувань, що задовольняють необхідну умову ефективності в задачі (37), (38). Саме збіжність алгоритмів до елементів множини $U^{*}$ - мета подальших теоретичних досліджень.

Для побудови алгоритмів використаємо опорну функцію $\sigma_{B}$ компактної множини $B \subseteq \mathbb{R}^{m} \backslash\{0\}$ такої, що $K^{*}=\operatorname{con}(\operatorname{conv} B)$. Для спрощення оцінок ми припустимо, що обрана множина $B$ має вигляд $\left\{k \in K^{*}:\|k\|_{\mathbb{R}^{m}}=1\right\}$. Тоді опорна функція $\sigma_{B}$ буде ліпшицевою з константою 1 . За допомогою цієї функції необхідну умову ефективності (39) можна записати у вигляді нерівності

$$
\begin{gathered}
\sigma_{B}\left(\begin{array}{c}
\left(\left(F^{\prime}(u)\right)^{*} p_{1}+D_{2} \Phi^{(1)}(y, u), v-u\right)_{V} \\
\cdots \\
\left(\left(F^{\prime}(u)\right)^{*} p_{m}+D_{2} \Phi^{(m)}(y, u), v-u\right)_{V}
\end{array}\right) \geq 0 \quad \forall v \in U, \\
\mathcal{L} y=F(u), \quad \mathcal{L}^{+} p_{i}=D_{1} \Phi^{(i)}(y, u), \quad i=\overline{1, m} .
\end{gathered}
$$

\section{Алгоритм 1. Метод лінеаризації.}

1. Обираємо початкове наближення $u_{0} \in U$. Покладемо $n=0$.

2. Знаходимо $\tilde{y}_{n} \in H_{1}:\left\|\tilde{y}_{n}-y_{n}\right\|_{H_{1}} \leq \delta_{n}^{\prime}$, де $y_{n} \in H_{1}-$ узагальнений розв'язок рівняння $\mathcal{L} y_{n}=F\left(u_{n}\right)$. 
3. Знаходимо $\overrightarrow{\tilde{p}}_{n}=\left(\tilde{p}_{n}^{1}, \tilde{p}_{n}^{2}, \ldots, \tilde{p}_{n}^{m}\right) \in\left(W_{2}\right)^{m}:\left\|\tilde{p}_{n}^{k}-\hat{p}_{n}^{k}\right\|_{W_{+}} \leq \delta_{n}^{\prime \prime}$, де $\hat{p}_{n}^{k} \in$ $W_{2}$ - розв'язки рівнянь $(k=\overline{1, m})$

$$
\begin{aligned}
& \mathcal{L}^{+} \hat{p}_{n}^{1}=D_{1} \Phi^{(1)}\left(\tilde{y}_{n}, u_{n}\right), \\
& \mathcal{L}^{+} \hat{p}_{n}^{m}=D_{1} \Phi^{(m)}\left(\tilde{y}_{n}, u_{n}\right) .
\end{aligned}
$$

4. Знаходимо $\bar{u}_{n} \in U-$ розв'язок екстремальної задачі

$$
\sigma_{B} \circ\left(\left(F^{\prime}\left(u_{n}\right)\right)^{*}{\overrightarrow{p_{n}}}_{n}+\Phi_{2}^{\prime}\left(\tilde{y}_{n}, u_{n}\right)\right)\left(u-u_{n}\right) \rightarrow \varepsilon_{n}-\inf _{u \in U} .
$$

5. Покладаємо $u_{n+1}=u_{n}+\rho_{n}\left(\bar{u}_{n}-u_{n}\right)$, де $\rho_{n} \in(0,1], n:=n+1$, i переходимо на крок 2.

Зауваження 6. Ідею побудови алгоритмів типу розглянутого було вперше висунуто у роботі [7]. У цитованій статті розглядалась задача

$$
f(x) \rightarrow \mathbb{R}_{+}^{m} \text { - min, } x \in \mathbb{R}^{n},
$$

де $f=\left(f_{1}, \ldots, f_{m}\right): \mathbb{R}^{n} \rightarrow \mathbb{R}^{m}$ - диференційовне відображення з похідною $f^{\prime}$, що задовольняє умову Ліпшиця. Для розв'язання (40) автори запропонували метод вигляду

$$
\begin{gathered}
x_{n+1}=x_{n}+\rho_{n}\left(\bar{x}_{n}-x_{n}\right), \\
\bar{x}_{n}=\arg \min \left\{\max _{k \in\{1, \ldots, m\}}\left(\operatorname{grad} f_{k}\left(x_{n}\right), x-x_{n}\right)+\frac{1}{2}\left\|x-x_{n}\right\|_{\mathbb{R}^{n}}^{2}\right\},
\end{gathered}
$$

де величина кроку $\rho_{n}$ обирається за правилом Арміхо. У алгоритмі 3 [7] множина $B$ має вигляд $\{(1,0, \ldots, 0),(0,1, \ldots, 0), \ldots,(0,0, \ldots, 1)\}$.

Має місце така теорема про збіжність алгоритму 1.

Теорема 15. Нехай $\rho_{n} \in(0,1], \rho_{n} \rightarrow 0, \sum_{n=0}^{\infty} \rho_{n}=+\infty, \varepsilon_{n} \rightarrow+0, \delta_{n}^{\prime} \rightarrow+0$, $\delta_{n}^{\prime \prime} \rightarrow+0$ i бункиіонал $\sigma_{B} \circ J$ приймае на множині $U^{*}$ не більш ніж зліченну кількість значенъ. Тодi всі граничні точки послідовності $\left(u_{n}\right)$ утворюють компактну зв'язну підмножину в $U^{*}$, а числова послідовність $\left(\sigma_{B}\left(J\left(u_{n}\right)\right)\right)$ має границю.

Доведення. Перевіримо виконання умов теореми про достатні умови збіжності ітераційних алгоритмів оптимізації [28]. Покладемо $W=\sigma_{B} \circ J$. За побудовою всі члени послідовності $\left(u_{n}\right)$ належать компакту $U$.

Маємо $\left\|u_{n+1}-u_{n}\right\|_{V}=\rho_{n}\left\|\bar{u}_{n}-u_{n}\right\|_{V} \leq \rho_{n} \operatorname{diam}(U) \rightarrow 0$ при $k \rightarrow \infty$.

Нехай $\left(u_{n_{k}}\right)$ - підпослідовність, що збігається до керування $u^{\prime} \notin U^{*}$. Покажемо, що існує $\delta_{0}>0$ таке, що для всіх $k$ та $\delta \in\left(0, \delta_{0}\right]$ :

$$
\tau_{k}=\min _{n>n_{k}}\left\{n:\left\|u_{n}-u_{n_{k}}\right\|_{V}>\delta\right\}<+\infty .
$$

Припустимо протилежне. Нехай для всіх $\delta_{0}>0$ існує таке $k_{0}=k_{0}\left(\delta_{0}\right) \in$ $\mathbb{N}$, що $\left\|u_{n}-u_{n_{k_{0}}}\right\|_{V} \leq \delta_{0}$ для всіх $n>n_{k_{0}}$. Тоді з нерівності трикутника маємо: $u_{n} \in B_{\delta_{0}}\left(u_{n_{k_{0}}}\right) \Rightarrow u_{n_{k}} \in B_{\delta_{0}}\left(u_{n_{k_{0}}}\right)$ для $k>k_{0} \Rightarrow u^{\prime} \in B_{\delta_{0}}\left(u_{n_{k_{0}}}\right) \Rightarrow$ $u_{n} \in B_{2 \delta_{0}}\left(u^{\prime}\right)$ для всіх $n>n_{k_{0}}$. 
Оскільки $u^{\prime} \notin U^{*}$, то існує число $\lambda>0$ таке, що

$$
\min _{u \in U} \sigma_{B}\left(J^{\prime}\left(u^{\prime}\right)\left(u-u^{\prime}\right)\right) \leq-2 \lambda<0 .
$$

Розглянемо приріст $W\left(u_{n+1}\right)-W\left(u_{n}\right)$ для $l>n_{k}>n_{k_{0}}$

$$
\begin{gathered}
W\left(u_{l}\right)-W\left(u_{n_{k}}\right)=\sigma_{B}\left(J\left(u_{l}\right)\right)-\sigma_{B}\left(J\left(u_{n_{k}}\right)\right) \leq \sigma_{B}\left(J\left(u_{l}\right)-J\left(u_{n_{k}}\right)\right)= \\
=\sigma_{B}\left(J\left(u_{l}\right)-J\left(u^{\prime}\right)-J\left(u_{n_{k}}\right)+J\left(u^{\prime}\right)\right) \leq \\
\leq \sigma_{B}\left(\begin{array}{c}
\left(D J^{(1)}\left(u^{\prime}\right), u_{l}-u_{n_{k}}\right)_{V} \\
\cdots \\
\left(D J^{(m)}\left(u^{\prime}\right), u_{l}-u_{n_{k}}\right)_{V}
\end{array}\right)+C_{0} \delta_{0}^{1+\gamma} \leq \\
\leq \sum_{n=n_{k}}^{l-1} \rho_{n} \sigma_{B}\left(J^{\prime}\left(u^{\prime}\right)\left(\bar{u}_{n}-u_{n}\right)\right)+C_{0} \delta_{0}^{1+\gamma} .
\end{gathered}
$$

Оцінимо зверху $\sigma_{B}\left(J^{\prime}\left(u^{\prime}\right)\left(\bar{u}_{n}-u_{n}\right)\right)$. Позначимо через $\bar{u}^{\prime} \in U$ розв'язок задачі мінімізації $\sigma_{B}\left(J^{\prime}\left(u^{\prime}\right)\left(u-u^{\prime}\right)\right) \rightarrow \inf _{u \in U}$. Маємо

$$
\begin{aligned}
& \sigma_{B}\left(J^{\prime}\left(u^{\prime}\right)\left(\bar{u}_{n}-u_{n}\right)\right) \leq \\
& \quad \leq \sigma_{B}\left(\left(J^{\prime}\left(u^{\prime}\right)-\widetilde{J^{\prime}\left(u_{n}\right)}\right)\left(\bar{u}_{n}-u_{n}\right)\right)+\sigma_{B}\left(\widetilde{J^{\prime}\left(u_{n}\right)}\left(\bar{u}_{n}-u_{n}\right)\right) \leq \\
& \quad \leq \sigma_{B}\left(\left(J^{\prime}\left(u^{\prime}\right)-\widetilde{J^{\prime}\left(u_{n}\right)}\right)\left(\bar{u}_{n}-u_{n}\right)\right)+\sigma_{B}\left(\widetilde{J^{\prime}\left(u_{n}\right)}\left(\bar{u}^{\prime}-u_{n}\right)\right)+\varepsilon_{n},
\end{aligned}
$$

де $\widetilde{J^{\prime}\left(u_{n}\right)}=\left(F^{\prime}\left(u_{n}\right)\right)^{*} \overrightarrow{\tilde{p}_{n}}+\Phi_{2}^{\prime}\left(\tilde{y}_{n}, u_{n}\right)$. Але

$$
\begin{aligned}
\sigma_{B}\left(\widehat{J^{\prime}\left(u_{n}\right)}\left(\bar{u}^{\prime}-u_{n}\right)\right) \leq \sigma_{B}\left(\left(\widetilde{J^{\prime}\left(u_{n}\right)}-J^{\prime}\left(u^{\prime}\right)\right)\left(\bar{u}^{\prime}-u_{n}\right)\right)+ \\
+\sigma_{B}\left(J^{\prime}\left(u^{\prime}\right)\left(\bar{u}^{\prime}-u^{\prime}\right)\right)+\sigma_{B}\left(J^{\prime}\left(u^{\prime}\right)\left(u^{\prime}-u_{n}\right)\right) \leq \\
\leq-2 \lambda+\sigma_{B}\left(\left(\widetilde{J^{\prime}\left(u_{n}\right)}-J^{\prime}\left(u^{\prime}\right)\right)\left(\bar{u}^{\prime}-u_{n}\right)\right)+\sigma_{B}\left(J^{\prime}\left(u^{\prime}\right)\left(u^{\prime}-u_{n}\right)\right) .
\end{aligned}
$$

Отже,

$$
\begin{aligned}
& \sigma_{B}\left(J^{\prime}\left(u^{\prime}\right)\left(\bar{u}_{n}-u_{n}\right)\right) \leq-2 \lambda+\sigma_{B}\left(\left(J^{\prime}\left(u^{\prime}\right)-\widetilde{J^{\prime}\left(u_{n}\right)}\right)\left(\bar{u}_{n}-u_{n}\right)\right)+ \\
& \quad+\sigma_{B}\left(\left(\widetilde{J^{\prime}\left(u_{n}\right)}-J^{\prime}\left(u^{\prime}\right)\right)\left(\bar{u}^{\prime}-u_{n}\right)\right)+\sigma_{B}\left(J^{\prime}\left(u^{\prime}\right)\left(u^{\prime}-u_{n}\right)\right)+\varepsilon_{n} .
\end{aligned}
$$

Нарешті отримаємо нерівність

$$
\begin{aligned}
& \sigma_{B}\left(J^{\prime}\left(u^{\prime}\right)\left(\bar{u}_{n}-u_{n}\right)\right) \leq \\
& \quad \leq-2 \lambda+2 \operatorname{diam}(U)\left\|\widetilde{J^{\prime}\left(u_{n}\right)}-J^{\prime}\left(u^{\prime}\right)\right\|+2\left\|J^{\prime}\left(u^{\prime}\right)\right\| \delta_{0}+\varepsilon_{n} .
\end{aligned}
$$

Оцінимо $\left\|\widetilde{J^{\prime}\left(u_{u}\right)}-J^{\prime}\left(u^{\prime}\right)\right\|$. Маємо

$$
\begin{gathered}
\left\|\widetilde{J^{\prime}\left(u_{n}\right)}-J^{\prime}\left(u^{\prime}\right)\right\| \leq\left\|\widetilde{J^{\prime}\left(u_{n}\right)}-J^{\prime}\left(u_{n}\right)\right\|+\left\|J^{\prime}\left(u_{n}\right)-J^{\prime}\left(u^{\prime}\right)\right\| \leq \\
\leq\left\|\widetilde{J^{\prime}\left(u_{n}\right)}-J^{\prime}\left(u_{n}\right)\right\|+C_{1}\left\|u_{n}-u^{\prime}\right\|_{V}^{\gamma} .
\end{gathered}
$$


Далі,

$$
\begin{aligned}
& \left\|\widetilde{J^{\prime}\left(u_{n}\right)}-J^{\prime}\left(u_{n}\right)\right\| \leq \\
& \leq\left\|\left(F^{\prime}\left(u_{n}\right)\right)^{*} \overrightarrow{\vec{p}_{n}}+\Phi_{2}^{\prime}\left(\tilde{y}_{n}, u_{n}\right)-\left(F^{\prime}\left(u_{n}\right)\right)^{*} \overrightarrow{p_{n}}-\Phi_{2}^{\prime}\left(y_{n}, u_{n}\right)\right\| \leq \\
& \leq\left\|F^{\prime}\left(u_{n}\right)\right\|\left\|\overrightarrow{\vec{p}_{n}}-\overrightarrow{p_{n}}\right\|_{\left(W_{2}\right)^{m}}+\left\|\Phi_{2}^{\prime}\left(\tilde{y}_{n}, u_{n}\right)-\Phi_{2}^{\prime}\left(y_{n}, u_{n}\right)\right\|_{V^{m}} \leq \\
& \leq C_{1}\left\|\overrightarrow{\hat{p}_{n}}-\overrightarrow{\hat{p}_{n}}\right\|_{\left(W_{2}\right)^{m}}+C_{1}\left\|\overrightarrow{\hat{p}_{n}}-\overrightarrow{p_{n}}\right\|_{\left(W_{2}\right)^{m}}+ \\
& \quad+C_{2}\left\|\tilde{y}_{n}-y_{n}\right\|_{H_{1}}^{\gamma} \leq C_{3} \delta_{n}^{\prime \prime}+C_{4}\left(\delta_{n}^{\prime}\right)^{\gamma}+ \\
& +C_{5}\left\|\Phi_{1}^{\prime}\left(\tilde{y}_{n}, u_{n}\right)-\Phi_{1}^{\prime}\left(y_{n}, u_{n}\right)\right\|_{\left(H_{1}^{-}\right)^{m} \leq C_{3} \delta_{n}^{\prime \prime}+C_{6}\left(\delta_{n}^{\prime}\right)^{\gamma},}
\end{aligned}
$$

де $\overrightarrow{p_{n}}=\left(p_{n}^{1}, \ldots, p_{n}^{m}\right) \in\left(W_{2}\right)^{m}$ - вектор розв'язків спряжених задач

$$
\mathcal{L}^{+} p_{n}^{i}=D_{1} \Phi^{(i)}\left(y_{n}, u_{n}\right), \quad i=\overline{1, m} .
$$

Отже,

$$
\begin{aligned}
\sigma_{B}\left(J^{\prime}\left(u^{\prime}\right)\left(\bar{u}_{n}-u_{n}\right)\right) & \leq \\
\leq & -2 \lambda+2 \operatorname{diam}(U)\left(C_{3} \delta_{n}^{\prime \prime}+C_{6}\left(\delta_{n}^{\prime}\right)^{\gamma}\right)+2\left\|J^{\prime}\left(u^{\prime}\right)\right\|_{V} \delta_{0}+\varepsilon_{n} .
\end{aligned}
$$

Обираючи достатньо мале $\delta_{0}>0$ та велике $k_{0}$, отримуємо $\left(n>n_{k_{0}}\right)$

$$
0<2 \operatorname{diam}(U)\left(C_{3} \delta_{n}^{\prime \prime}+C_{6}\left(\delta_{n}^{\prime}\right)^{\gamma}\right)+2\left\|J^{\prime}\left(u^{\prime}\right)\right\|_{V} \delta_{0}+\varepsilon_{n}<\lambda .
$$

Звідки

$$
\sigma_{B}\left(J^{\prime}\left(u^{\prime}\right)\left(\bar{u}_{n}-u_{n}\right)\right) \leq-\lambda, \quad n>n_{k_{0}}
$$

Остаточно маємо

$$
W\left(u_{l}\right)-W\left(u_{n_{k}}\right) \leq-\lambda \sum_{n=n_{k}}^{l-1} \rho_{n}+C_{0} \delta_{0}^{1+\gamma}, \quad l>n_{k} \geq n_{k_{0}} .
$$

Після граничного переходу в нерівності при $m \rightarrow \infty\left(\sum_{n=n_{k}}^{\infty} \rho_{n}=+\infty\right)$ отримаємо протиріччя з обмеженістю знизу на множині $U$ функціонала $W$.

Отже, існує $\delta_{0}>0$ таке, що для всіх $k$ i $\delta \in\left(0, \delta_{0}\right]$ :

$$
\tau_{k}=\min _{n>n_{k}}\left\{n:\left\|u_{n}-u_{n_{k}}\right\|_{V}>\delta\right\}<+\infty .
$$

Але обираючи достатньо мале $\delta_{0}>0$ та велике $k_{0}$, можна повторити доведення оцінки (41) для $n_{k} \leq n \leq \tau_{k}$. 3 іншого боку,

$$
\delta_{0}<\left\|u_{\tau_{k}}-u_{n_{k}}\right\|_{V} \leq \sum_{p=n_{k}}^{\tau_{k}-1}\left\|u_{p+1}-u_{p}\right\|_{V} \leq \operatorname{diam}(U) \sum_{p=n_{k}}^{\tau_{k}-1} \rho_{p} .
$$

Тому

$$
\sum_{p=n_{k}}^{\tau_{k}-1} \rho_{p}>\frac{\delta_{0}}{\operatorname{diam}(U)}
$$


Ураховуючи останню нерівність в (41), маємо

$$
W\left(u_{\tau_{k}}\right)<W\left(u_{n_{k}}\right)-\frac{\lambda \delta_{0}}{\operatorname{diam}(U)}+C \delta_{0}^{1+\gamma} .
$$

Звідки $\lim \sup _{k \rightarrow \infty} W\left(u_{\tau_{k}}\right)<\lim _{k \rightarrow \infty} W\left(u_{n_{k}}\right)$. Отже, умови теореми про збіжність виконуються, а тому, граничні точки послідовності $\left(u_{n}\right)$ утворюють компактну зв'язну підмножину $U^{*}$ і послідовність чисел $\sigma_{B}\left(J\left(u_{n}\right)\right)$ має границю.

Розглянемо метод з довірчою областю для задачі (37), (38). Зафіксуємо дві числові послідовності $\left(\alpha_{n}\right)$ і $\left(\beta_{n}\right)$ такі, що $0<\alpha_{n} \leq \beta_{n}(n \in \mathbb{N})$, та послідовність замкнених опуклих множин $M_{n} \subseteq V$ простої структури таку, що

$$
\left\{v \in V:\|v\|_{V} \leq \alpha_{n}\right\} \subseteq M_{n} \subseteq\left\{v \in V:\|v\|_{V} \leq \beta_{n}\right\} .
$$

\section{Алгоритм 2. Метод з довірчою областю.}

1. Обираємо початкове наближення $u_{0} \in U$. Покладемо $n=0$.

2. Знаходимо $y_{n} \in H_{1}$ - розв'язок рівняння $\mathcal{L} y_{n}=F\left(u_{n}\right)$.

3. Знаходимо спряжені стани $\overrightarrow{p_{n}}=\left(p_{n}^{1}, p_{n}^{2}, \ldots, p_{n}^{m}\right) \in\left(W_{2}\right)^{m}$

$$
\begin{gathered}
\mathcal{L}^{+} p_{n}^{1}=D_{1} \Phi^{(1)}\left(y_{n}, u_{n}\right), \\
\mathcal{L}^{+} p_{n}^{m}=D_{1} \Phi^{(m)}\left(y_{n}, u_{n}\right) .
\end{gathered}
$$

4. Знаходимо $\bar{u}_{n} \in U-$ розв'язок екстремальної задачі

$$
\sigma_{B} \circ\left(\left(F^{\prime}\left(u_{n}\right)\right)^{*} \overrightarrow{p_{n}}+\Phi_{2}^{\prime}\left(y_{n}, u_{n}\right)\right)\left(u-u_{n}\right) \rightarrow \varepsilon_{n}-\inf _{u \in U \cap\left(u_{n}+M_{n}\right)} .
$$

5. Покладаємо $u_{n+1}=\bar{u}_{n}, n:=n+1$ і переходимо на крок 2 .

Відносно послідовності $\left(u_{n}\right)$, породженої алгоритмом 2 , справджується такий факт.

Теорема 16. Нехай

$$
\varepsilon_{n}>0, k \cdot \alpha_{n} \geq \beta_{n} \geq \alpha_{n}>0, \sum_{n=0}^{\infty} \alpha_{n}=+\infty, \frac{\beta_{n}^{1+\gamma}}{\alpha_{n}} \rightarrow 0, \frac{\varepsilon_{n}}{\alpha_{n}} \rightarrow 0
$$

$i$ множина $\left(\sigma_{B} \circ J\right)\left(U^{*}\right)$ ніде не щільна. Тоді всі граничні точки послідовності $\left(u_{n}\right)$ утворюють компактну зв'язну підмножсиу в $U^{*}$, а числова послідовність $\left(\left(\sigma_{B} \circ J\right)\left(u_{n}\right)\right)$ мае гранищю.

Доведення. Використаємо загальну теорему про збіжність алгоритмів [28]. За побудовою всі члени послідовності $\left(u_{n}\right)$ належать компакту $U$. Далі

$$
\left\|u_{n+1}-u_{n}\right\|_{V}=\left\|\bar{u}_{n}-u_{n}\right\|_{V} \leq \beta_{n} \rightarrow 0 \text { при } n \rightarrow \infty .
$$

Нехай $\left(u_{n_{k}}\right)$ - підпослідовність, що збігається до керування $u^{\prime} \notin U^{*}$. Покажемо, що існує таке $\delta_{0}>0$, що для всіх $k$ та $\delta \in\left(0, \delta_{0}\right]$ :

$$
\tau_{k}=\min _{n>n_{k}}\left\{n:\left\|u_{n}-u_{n_{k}}\right\|_{V}>\delta\right\}<+\infty .
$$


Від супротивного. Нехай для всіх $\delta_{0}>0$ існуе $k_{0}=k_{0}\left(\delta_{0}\right)$ таке, що $\| u_{n}-$ $u_{n_{k_{0}}} \|_{V} \leq \delta_{0}$ для всіх $n>n_{k_{0}}$. Тоді з нерівності трикутника випливає $u_{n} \in$ $B_{2 \delta_{0}}\left(u^{\prime}\right)$ для всіх $n>n_{k_{0}}$.

Оцінимо приріст $\sigma_{B}\left(J\left(u_{n+1}\right)\right)-\sigma_{B}\left(J\left(u_{n}\right)\right)$. Маємо

$$
\begin{aligned}
& \sigma_{B}\left(J\left(u_{n+1}\right)\right)-\sigma_{B}\left(J\left(u_{n}\right)\right) \leq \\
& \leq \sigma_{B}\left(J^{\prime}\left(u_{n}\right)\left(u_{n+1}-u_{n}\right)\right)+\frac{C_{1}}{\gamma+1}\left\|u_{n+1}-u_{n}\right\|_{V}^{\gamma+1} \leq \\
& \quad \leq \min _{u \in U \cap\left(u_{n}+M_{n}\right)} \sigma_{B}\left(J^{\prime}\left(u_{n}\right)\left(u-u_{n}\right)\right)+\varepsilon_{n}+\frac{C_{1}}{\gamma+1} \beta_{n}^{\gamma+1} .
\end{aligned}
$$

Оскільки $u^{\prime} \notin U^{*}$, то існує число $\lambda>0$ таке, що

$$
\min _{u \in U} \sigma_{B}\left(J^{\prime}\left(u^{\prime}\right)\left(u-u^{\prime}\right)\right) \leq-2 \lambda<0 .
$$

Із неперервності функціонала $\xi \mapsto \min _{u \in U} \sigma_{B}\left(J^{\prime}(\xi)(u-\xi)\right)$ випливає існування $\delta>0$ такого, що для довільної точки $u^{\prime \prime} \in O_{\delta}\left(u^{\prime}\right) \cap U$ виконується нерівність

$$
\min _{u \in U} \sigma_{B}\left(J^{\prime}\left(u^{\prime \prime}\right)\left(u-u^{\prime \prime}\right)\right) \leq-\lambda<0 .
$$

Візьмемо $2 \delta_{0}<\delta$, тоді $u_{n} \in B_{2 \delta_{0}}\left(u^{\prime}\right) \subseteq O_{\delta}\left(u^{\prime}\right)$. Позначимо через $\overline{\bar{u}}_{n} \in U$ розв'язок задачі мінімізації

$$
\sigma_{B}\left(J^{\prime}\left(u_{n}\right)\left(u-u_{n}\right)\right) \rightarrow \inf _{u \in U} .
$$

Нехай $\overline{\bar{u}}_{n} \in u_{n}+M_{n}$. Тоді з (42) випливає нерівність

$$
\sigma_{B}\left(J\left(u_{n+1}\right)\right)-\sigma_{B}\left(J\left(u_{n}\right)\right) \leq-\lambda+\varepsilon_{n}+\frac{C_{1}}{\gamma+1} \beta_{n}^{\gamma+1} .
$$

Існує $n^{\prime}>n_{k_{0}}$ таке, що для всіх $n \geq n^{\prime}$ :

$$
\sigma_{B}\left(J\left(u_{n+1}\right)\right)-\sigma_{B}\left(J\left(u_{n}\right)\right) \leq-\frac{\lambda}{2} .
$$

Якщо $\overline{\bar{u}}_{n} \notin u_{n}+M_{n}$, то

$$
\begin{aligned}
\sigma_{B}\left(J\left(u_{n+1}\right)\right)-\sigma_{B}\left(J\left(u_{n}\right)\right) & \leq \\
\leq \sigma_{B}\left(J^{\prime}\left(u_{n}\right)\right. & \left.\left(\phi_{n}-u_{n}\right)\right)+\varepsilon_{n}+\frac{C_{1}}{\gamma+1} \beta_{n}^{\gamma+1} \leq \\
& \leq \eta_{n} \sigma_{B}\left(J^{\prime}\left(u_{n}\right)\left(\overline{\bar{u}}_{n}-u_{n}\right)\right)+\varepsilon_{n}+\frac{C_{1}}{\gamma+1} \beta_{n}^{\gamma+1},
\end{aligned}
$$

де $\eta_{n}=\max \left\{\eta>0: \eta\left(\overline{\bar{u}}_{n}-u_{n}\right) \in M_{n}\right\}, \phi_{n}=u_{n}+\eta_{n}\left(\overline{\bar{u}}_{n}-u_{n}\right)$. У цьому випадку приріст оцінюється так:

$$
\sigma_{B}\left(J\left(u_{n+1}\right)\right)-\sigma_{B}\left(J\left(u_{n}\right)\right) \leq-\eta_{n} \lambda+\varepsilon_{n}+\frac{C_{1}}{\gamma+1} \beta_{n}^{\gamma+1} .
$$


Оскільки $1>\mu_{n} \geq \frac{\alpha_{n}}{\left\|\overline{\bar{u}}_{n}-u_{n}\right\|_{V}}$, то

$$
\begin{aligned}
\sigma_{B}\left(J\left(u_{n+1}\right)\right)-\sigma_{B} & \left(J\left(u_{n}\right)\right) \leq-\frac{\lambda \alpha_{n}}{\left\|\overline{\bar{u}}_{n}-u_{n}\right\|_{V}}+\varepsilon_{n}+\frac{C_{1}}{\gamma+1} \beta_{n}^{\gamma+1} \leq \\
\leq & -\frac{\lambda}{\operatorname{diam}(U)} \alpha_{n}+\varepsilon_{n}+\frac{C_{1}}{\gamma+1} \beta_{n}^{\gamma+1}= \\
& =-\frac{\lambda}{\operatorname{diam}(U)} \alpha_{n}\left(1-\frac{\operatorname{diam}(U)}{\lambda}\left(\frac{\varepsilon_{n}}{\alpha_{n}}+\frac{C_{1}}{\gamma+1} \frac{\beta_{n}^{\gamma+1}}{\alpha_{n}}\right)\right),
\end{aligned}
$$

звідки випливає існування $n^{\prime \prime}>n_{k_{0}}$ такого, що для всіх $n \geq n^{\prime \prime}$

$$
\sigma_{B}\left(J\left(u_{n+1}\right)\right)-\sigma_{B}\left(J\left(u_{n}\right)\right) \leq-\frac{\lambda}{2 \cdot \operatorname{diam}(U)} \alpha_{n} \leq-\frac{\lambda}{2 \cdot k \cdot \operatorname{diam}(U)} \beta_{n} .
$$

Існує $n^{\prime \prime \prime} \in \mathbb{N}$ таке, що $\beta_{n}<\lambda / 2$ для всіх $n \geq n^{\prime \prime \prime}$. Отже,

$$
\sigma_{B}\left(J\left(u_{n+1}\right)\right)-\sigma_{B}\left(J\left(u_{n}\right)\right) \leq-\min \left\{1, \frac{\lambda}{2 \cdot k \cdot \operatorname{diam}(U)}\right\} \beta_{n},
$$

для $n>\tilde{n}=\max \left\{n^{\prime}, n^{\prime \prime}, n^{\prime \prime \prime}\right\}>n_{k_{0}}$.

Остаточно маємо

$$
\begin{aligned}
& \sigma_{B}\left(J\left(u_{n}\right)\right)-\sigma_{B}\left(J\left(u_{n_{k}}\right)\right) \leq \\
& \leq-\min \left\{1, \frac{\lambda}{2 \cdot k \cdot \operatorname{diam}(U)}\right\} \sum_{p=n_{k}}^{n-1} \beta_{p}, \quad n>n_{k}>\tilde{n} .
\end{aligned}
$$

Здійснивши граничний перехід в нерівності (43) при $n \rightarrow \infty$ та урахувавши $\sum_{p=1}^{\infty} \beta_{p}=+\infty$, отримаємо протиріччя з обмеженістю на компактній множині $U$ неперервного функціонала $\sigma_{B} \circ J$. Отже, існуе $\delta_{0}>0$ таке, що для всіх $k$ та $\delta \in\left(0, \delta_{0}\right]: \tau_{k}=\min _{n>n_{k}}\left\{n:\left\|u_{n}-u_{n_{k}}\right\|_{V}>\delta\right\}<+\infty$. Але обираючи достатньо мале $\delta_{0}>0$ та велике $k_{0}$, можна повторити доведення нерівності (41) для $n_{k} \leq n \leq \tau_{k}$. 3 іншого боку,

$$
\delta_{0}<\left\|u_{\tau_{k}}-u_{n_{k}}\right\|_{V} \leq \sum_{p=n_{k}}^{\tau_{k}-1}\left\|u_{p+1}-u_{p}\right\|_{V} \leq \sum_{p=n_{k}}^{\tau_{k}-1} \beta_{p} .
$$

Ураховуючи останню нерівність в (43), маємо

Звідки маємо

$$
\sigma_{B}\left(J\left(u_{\tau_{k}}\right)\right)<\sigma_{B}\left(J\left(u_{n_{k}}\right)\right)-\min \left\{1, \frac{\lambda}{2 \cdot k \cdot \operatorname{diam}(U)}\right\} \delta_{0} .
$$

$$
\limsup _{k \rightarrow \infty} \sigma_{B}\left(J\left(u_{\tau_{k}}\right)\right)<\lim _{k \rightarrow \infty} \sigma_{B}\left(J\left(u_{n_{k}}\right)\right) .
$$

Покладемо $W=\sigma_{B} \circ J$. Умови теореми про збіжність ітераційних алгоритмів виконуються, а отже, послідовність $\left(u_{n}\right)$ має сформульовані властивості.

Розглянемо ще один алгоритм розв'язання задачі (37), (38) - векторний аналог методу проекції градієнта. 


\section{Алгоритм 3. Послідовно-квадратичного метод.}

1. Обираємо початкове наближення $u_{0} \in U$. Покладемо $n=0$.

2. Знаходимо $y_{n} \in H_{1}-$ узагальнений розв'язок рівняння

$$
\mathcal{L} y_{n}=F\left(u_{n}\right) .
$$

3. Знаходимо спряжені стани $\overrightarrow{p_{n}}=\left(p_{n}^{1}, p_{n}^{2}, \ldots, p_{n}^{m}\right) \in\left(W_{2}\right)^{m}$

$$
\begin{aligned}
& \mathcal{L}^{+} p_{n}^{1}=D_{1} \Phi^{(1)}\left(y_{n}, u_{n}\right), \\
& \mathcal{L}^{+} p_{n}^{m}=D_{1} \Phi^{(m)}\left(y_{n}, u_{n}\right) .
\end{aligned}
$$

4. Знаходимо $\bar{u}_{n} \in U-$ розв'язок екстремальної задачі

$$
\sigma_{B} \circ\left(\left(F^{\prime}\left(u_{n}\right)\right)^{*} \overrightarrow{p_{n}}+\Phi_{2}^{\prime}\left(y_{n}, u_{n}\right)\right)\left(u-u_{n}\right)+\frac{1}{2 \alpha_{n}}\left\|u-u_{n}\right\|_{V}^{2} \rightarrow \varepsilon_{n}-\inf _{u \in U} .
$$

5. Покладаємо $u_{n+1}=u_{n}+\rho_{n}\left(\bar{u}_{n}-u_{n}\right)$, де $\rho_{n} \in(0,1], n:=n+1 \mathrm{i}$ переходимо на крок 2.

Має місце теорема.

Теорема 17. Нехай $\rho_{n} \in[\underline{\rho}, \bar{\rho}] \subseteq(0,1], \alpha_{n} \rightarrow 0, \sum_{n=0}^{\infty} \alpha_{n}=+\infty, \varepsilon_{n}>0$, $\frac{\varepsilon_{n}}{\alpha_{n}} \rightarrow 0$. Якщо функиіонал $\sigma_{B} \circ J$ приймае на множині $U^{*}$ не більш ніж зліченну кількість значень, то всі граничні точки послідовності $\left(u_{n}\right)$, породженої алгоритмом 3, утворюють компактну зв'язну підмножину в $U^{*}$, а послідовність чисел $\sigma_{B}\left(J\left(u_{n}\right)\right)$ мае границю.

Доведення. Доведення подібне до попередніх. Перевіримо виконання умов теореми про збіжність ітераційних алгоритмів [28]. За побудовою усі члени послідовності $\left(u_{n}\right)$ належать компакту $U$.

Розглянемо підпослідовність $\left(u_{n_{k}}\right)$ таку, що $u_{n_{k}} \rightarrow u^{*} \in U^{*}$ при $k \rightarrow \infty$. Оцінимо $\left\|u_{n_{k}+1}-u_{n_{k}}\right\|_{V}$. Функціонал

$$
\begin{aligned}
u \mapsto G_{n_{k}}(u)=\sigma_{B} \circ\left(\left(F^{\prime}\left(u_{n_{k}}\right)\right)^{*} \overrightarrow{p_{n_{k}}}+\Phi_{2}^{\prime}\left(y_{n_{k}}, u_{n_{k}}\right)\right) & \left(u-u_{n_{k}}\right)+ \\
& +\frac{1}{2 \alpha_{n_{k}}}\left\|u-u_{n_{k}}\right\|_{V}^{2}
\end{aligned}
$$

сильно опуклий $\left(\frac{1}{2 \alpha_{n_{k}}}>0-\right.$ стала сильної опуклості). Покладемо

$$
\tilde{u}_{n_{k}}=\arg \min _{u \in U} G_{n_{k}}(u) \text {. }
$$

Маємо

Отже,

$$
\frac{1}{2 \alpha_{n_{k}}}\left\|\bar{u}_{n_{k}}-\tilde{u}_{n_{k}}\right\|_{V}^{2} \leq G\left(\bar{u}_{n_{k}}\right)-G\left(\tilde{u}_{n_{k}}\right) \leq \varepsilon_{n_{k}} .
$$

$$
\begin{aligned}
\left\|u_{n_{k}+1}-u_{n_{k}}\right\|_{V} & =\rho_{n_{k}}\left\|\bar{u}_{n_{k}}-u_{n_{k}}\right\|_{V} \leq \bar{\rho}\left\|\bar{u}_{n_{k}}-u_{n_{k}}\right\|_{V} \leq \\
& \leq\left\|\bar{u}_{n_{k}}-\tilde{u}_{n_{k}}\right\|_{V}+\left\|\tilde{u}_{n_{k}}-u_{n_{k}}\right\|_{V} \leq \sqrt{2 \alpha_{n_{k}} \varepsilon_{n_{k}}}+C_{0} \alpha_{n_{k}} \rightarrow 0
\end{aligned}
$$

при $k \rightarrow \infty$, де $C_{0}=2 \sup _{u \in U}\left\|J^{\prime}(u)\right\|_{V}<+\infty$. 
Нехай $\left(u_{n_{k}}\right)$ - підпослідовність, що збігається до керування $u^{\prime} \notin U^{*}$. Покажемо, що існує таке $\delta_{0}>0$, що для всіх $k$ та $\delta \in\left(0, \delta_{0}\right]$ :

$$
\tau_{k}=\min _{n>n_{k}}\left\{n:\left\|u_{n}-u_{n_{k}}\right\|_{V}>\delta\right\}<+\infty .
$$

Від супротивного. Нехай для всіх $\delta_{0}>0$ існує $k_{0}=k_{0}\left(\delta_{0}\right)$ таке, що $\| u_{n}-$ $u_{n_{k_{0}}} \|_{V} \leq \delta_{0}$ для всіх $n>n_{k_{0}}$. Тоді $u_{n} \in B_{2 \delta_{0}}\left(u^{\prime}\right)$ для всіх $n>n_{k_{0}}$.

Оцінимо приріст $\sigma_{B}\left(J\left(u_{n+1}\right)\right)-\sigma_{B}\left(J\left(u_{n}\right)\right)$. Маємо

$$
\begin{gathered}
\sigma_{B}\left(J\left(u_{n+1}\right)\right)-\sigma_{B}\left(J\left(u_{n}\right)\right) \leq \sigma_{B}\left(J\left(u_{n+1}\right)-J\left(u_{n}\right)\right) \leq \\
\leq \sigma_{B}\left(J^{\prime}\left(u_{n}\right)\left(u_{n+1}-u_{n}\right)\right)+\frac{C_{1}}{\gamma+1}\left\|u_{n+1}-u_{n}\right\|_{V}^{\gamma+1}= \\
=\rho_{n} \sigma_{B}\left(J^{\prime}\left(u_{n}\right)\left(\bar{u}_{n}-u_{n}\right)\right)+\frac{C_{1}}{\gamma+1} \rho_{n}^{\gamma+1}\left\|\bar{u}_{n}-u_{n}\right\|_{V}^{\gamma+1}= \\
=\rho_{n}\left\{\sigma_{B}\left(J^{\prime}\left(u_{n}\right)\left(\bar{u}_{n}-u_{n}\right)\right)+\frac{1}{2 \alpha_{n}}\left\|\bar{u}_{n}-u_{n}\right\|_{V}^{2}\right\}+ \\
\quad+\frac{C_{1}}{\gamma+1} \rho_{n}^{\gamma+1}\left\|\bar{u}_{n}-u_{n}\right\|_{V}^{\gamma+1}-\frac{\rho_{n}}{2 \alpha_{n}}\left\|\bar{u}_{n}-u_{n}\right\|_{V}^{2} \leq \\
\quad \leq \rho_{n}\left\{\varepsilon_{n}+\min _{u \in U} G_{n}(u)\right\}+\frac{C_{1}}{\gamma+1} \rho_{n}^{\gamma+1}\left\|\bar{u}_{n}-u_{n}\right\|_{V}^{\gamma+1} .
\end{gathered}
$$

Оскільки $u^{\prime} \notin U^{*}$, то існує таке $\lambda>0$, що

$$
\min _{u \in U} \sigma_{B}\left(J^{\prime}\left(u^{\prime}\right)\left(u-u^{\prime}\right)\right) \leq-2 \lambda<0 .
$$

Далі існуе $\delta>0$ таке, що для довільної точки $u^{\prime \prime} \in O_{\delta}\left(u^{\prime}\right)$ виконується нерівність

$$
\min _{u \in U} \sigma_{B}\left(J^{\prime}\left(u^{\prime \prime}\right)\left(u-u^{\prime \prime}\right)\right) \leq-\lambda<0 .
$$

Візьмемо $2 \delta_{0}<\delta$, тоді $u_{n} \in B_{2 \delta_{0}}\left(u^{\prime}\right) \subseteq O_{\delta}\left(u^{\prime}\right)$. Позначимо через $\overline{\bar{u}}_{n} \in U$ розв'язок задачі мінімізації

$$
\sigma_{B}\left(J^{\prime}\left(u_{n}\right)\left(u-u_{n}\right)\right) \rightarrow \inf _{u \in U} .
$$

Для $\overline{\bar{u}}_{n} \in U$ виконується нерівність

$$
-\left\|J^{\prime}\left(u_{n}\right)\right\| \cdot\left\|\overline{\bar{u}}_{n}-u_{n}\right\|_{V} \leq \sigma_{B}\left(J^{\prime}\left(u_{n}\right)\left(\overline{\bar{u}}_{n}-u_{n}\right)\right) \leq-\lambda<0,
$$

тобто,

$$
\frac{1}{\left\|\overline{\bar{u}}_{n}-u_{n}\right\|_{V}} \leq \frac{\left\|J^{\prime}\left(u_{n}\right)\right\|}{\lambda} \leq \frac{C_{0}}{\lambda}
$$

Обираючи достатньо велике $k_{0}$, ми можемо досягти для всіх $n>n_{k_{0}}$ виконання нерівності $\alpha_{n} \leq \lambda / C_{0}^{2}$. Розглянемо точку $u_{n}^{\prime \prime}=u_{n}+\mu_{n}\left(\overline{\bar{u}}_{n}-u_{n}\right)$, де $\mu_{n}=\frac{\lambda \alpha_{n}}{\left\|\overline{\bar{u}}_{n}-u_{n}\right\|_{V}^{2}} \in(0,1]$. Очевидно, що $u_{n}^{\prime \prime} \in U$. Підставимо точку $u_{n}^{\prime \prime} \in U \mathrm{y}$ 
праву частину нерівності (44). Отримаємо такий ланцюжок оцінок

$$
\begin{gathered}
\sigma_{B}\left(J\left(u_{n+1}\right)\right)-\sigma_{B}\left(J\left(u_{n}\right)\right) \leq \\
\leq \rho_{n}\left\{\varepsilon_{n}+\sigma_{B}\left(J^{\prime}\left(u_{n}\right)\left(u_{n}^{\prime \prime}-u_{n}\right)\right)+\frac{1}{2 \alpha_{n}}\left\|u_{n}^{\prime \prime}-u_{n}\right\|_{V}^{2}\right\}+ \\
+\frac{C_{1}}{\gamma+1} \rho_{n}^{\gamma+1}\left\|\bar{u}_{n}-u_{n}\right\|_{V}^{\gamma+1}= \\
=\rho_{n}\left\{\varepsilon_{n}+\mu_{n} \sigma_{B}\left(J^{\prime}\left(u_{n}\right)\left(\overline{\bar{u}}_{n}-u_{n}\right)\right)+\frac{\mu_{n}^{2}}{2 \alpha_{n}}\left\|\overline{\bar{u}}_{n}-u_{n}\right\|_{V}^{2}\right\}+ \\
+\frac{C_{1}}{\gamma+1} \rho_{n}^{\gamma+1}\left\|\bar{u}_{n}-u_{n}\right\|_{V}^{\gamma+1} \leq \\
\leq \rho_{n}\left\{\varepsilon_{n}-\mu_{n} \lambda+\frac{\mu_{n}^{2}}{2 \alpha_{n}}\left\|\overline{\bar{u}}_{n}-u_{n}\right\|_{V}^{2}\right\}+\frac{C_{1}}{\gamma+1}\left(\alpha_{n} \sqrt{\frac{2 \varepsilon_{n}}{\alpha_{n}}}+C_{0} \alpha_{n}\right)^{\gamma+1}= \\
=\rho_{n}\left\{\varepsilon_{n}-\frac{\lambda^{2} \alpha_{n}}{2\left\|\overline{\bar{u}}_{n}-u_{n}\right\|_{V}^{2}}\right\}+\frac{C_{1}}{\gamma+1}\left(\alpha_{n} \sqrt{\frac{2 \varepsilon_{n}}{\alpha_{n}}}+C_{0} \alpha_{n}\right)^{\gamma+1} \leq \\
\leq-\frac{\rho}{2}\left(\frac{\lambda}{\operatorname{diam}(U)}\right)^{2} \alpha_{n}+\varepsilon_{n}+\frac{C_{1}}{\gamma+1} \alpha_{n}^{\gamma+1}\left(\sqrt{\frac{2 \varepsilon_{n}}{\alpha_{n}}}+C_{0}\right)^{\gamma+1} .
\end{gathered}
$$

Оскільки $\alpha_{n} \rightarrow 0, \varepsilon_{n} \rightarrow 0$ i $\frac{\varepsilon_{n}}{\alpha_{n}} \rightarrow 0$, то, обираючи достатньо мале $\delta_{0}>0$ та велике $k_{0}$, отримуємо

$$
\sigma_{B}\left(J\left(u_{n+1}\right)\right)-\sigma_{B}\left(J\left(u_{n}\right)\right) \leq-\frac{\rho \lambda^{2} \alpha_{n}}{4(\operatorname{diam}(U))^{2}}, \quad n>n_{k_{0}} .
$$

Остаточно маємо

$$
\sigma_{B}\left(J\left(u_{n}\right)\right)-\sigma_{B}\left(J\left(u_{n_{k}}\right)\right) \leq-\frac{\underline{\rho} \lambda^{2}}{4(\operatorname{diam}(U))^{2}} \sum_{p=n_{k}}^{n-1} \alpha_{p}, n>n_{k}>n_{k_{0}} .
$$

Здійснивши граничний перехід у нерівності (45) при $n \rightarrow \infty$ та врахувавши $\sum_{p=n_{k}}^{\infty} \alpha_{p}=+\infty$, отримаємо протиріччя з обмеженістю на компактній множині $U$ неперервного функціонала $\sigma_{B} \circ J$. Отже, існує $\delta_{0}>0$ таке, що для всіх $k$ та $\delta \in\left(0, \delta_{0}\right]$

$$
\tau_{k}=\min _{n>n_{k}}\left\{n:\left\|u_{n}-u_{n_{k}}\right\|_{V}>\delta\right\}<+\infty .
$$

Але обираючи достатньо мале $\delta_{0}>0$ та велике $k_{0}$, можна повторити доведення нерівності (45) для $n_{k} \leq n \leq \tau_{k}$. 3 іншого боку,

$$
\begin{aligned}
& \delta_{0}<\left\|u_{\tau_{k}}-u_{n_{k}}\right\|_{V} \leq \sum_{p=n_{k}}^{\tau_{k}-1} \| u_{p+1}- u_{p} \|_{V} \leq \\
& \leq \sum_{p=n_{k}}^{\tau_{k}-1}\left(\sqrt{2 \alpha_{p} \varepsilon_{p}}+C_{0} \alpha_{p}\right) \leq C_{2} \sum_{p=n_{k}}^{\tau_{k}-1} \alpha_{p} .
\end{aligned}
$$


Тому $\sum_{p=n_{k}}^{\tau_{k}-1} \alpha_{p}>\delta_{0} / C_{2}>0$. Ураховуючи останню нерівність в (38), маємо

$$
\sigma_{B}\left(J\left(u_{\tau_{k}}\right)\right)-\sigma_{B}\left(J\left(u_{n_{k}}\right)\right)<-\underline{\rho} \lambda^{2} \delta_{0} / 4 C_{2}(\operatorname{diam}(U))^{2} .
$$

Звідки

$$
\limsup _{k \rightarrow \infty} \sigma_{B}\left(J\left(u_{\tau_{k}}\right)\right)<\lim _{k \rightarrow \infty} \sigma_{B}\left(J\left(u_{n_{k}}\right)\right) .
$$

Покладемо $W=\sigma_{B} \circ J$. Умови теореми про збіжність ітераційних алгоритмів виконуються.

Зауваження 7. Відмітимо, що в запропонованих методах є один момент, який має бути вирішений: на кожному ітераційному кроці слід ефективно розв'язувати негладку задачу кусково-лінійної чи кусково-квадратичної оптимізації.

\section{ЗАКЛЮЧНІ ЗАУВАЖЕННЯ}

У роботі розвинена теорія існування та необхідних умов оптимальності для задач оптимального керування з векторним критерієм якості системами, що описуються рівняннями математичної фізики з узагальненими впливами. Увагу приділено побудові і дослідженню збіжності методів розв'язання задач векторної оптимізації лінійних розподілених систем. Досліджено поняття $(K, e, \varepsilon)$-апроксимаційної ефективності. Грунтуючись на векторному варіанті класичного варіаційного принципу Екланда, доведено умови $(K, e, \varepsilon)$-апроксимаційної ефективності допустимих керувань у вигляді варіаційних включень. Запропоновано методи розв'язання задач векторної оптимізації лінійних розподілених систем з узагальненим керуванням. Доведено збіжність алгоритмів із похибками в ітераційних підзадачах.

Робота виконана за фінансової підтримки МОН України (проєкт «Математичне моделювання та оптимізація динамічних систем для оборони, екології та медицини», 0219U008403, 2019-2021рр.) та НАН України (проєкт «Нові методи дослідження коректності та розв'язання задач дискретної оптимізації, варіаційних нерівностей та їх застосування», 0119U101608, 2019-2020 pp.).

\section{ЛiтеРАТУРА}

1. Салуквадзе М. Е. Задачи векторной оптимизации в теории управления. Тбилиси: Мецниереба, 1975. 201 с.

2. Stadler W. A Survey of Multicriteria Optimization or the Vector Maximum Problem, Part I: 1776-1960. Journal of Optimization Theory and Applications. 1979. Vol. 29. No. 1. P. 1-52.

3. Dauer J. P., Stadler W. A Survey of Vector Optimization in Infinite-Dimensional Spaces, Part 2. Journal of Optimization Theory and Applications. 1986. Vol. 51. No. 2. P. 205-241.

4. Гороховик В. В. Выпуклые и негладкие задачи векторной оптимизации. Минск: Навука і тэхніка, 1990. 238 с.

5. Gopfert A., Tammer Chr., Riahi H., Zalinescu C. Variational Methods in Partially Ordered Spaces. New York-Berlin-Heidelberg: Springer-Verlag, 2003. 350 p. 
6. Подиновский В. В., Ногин В. Д. Парето-оптимальные решения многокритериальных задач. 2-е изд., испр. и доп. Москва: ФИЗМАТЛИТ, 2007. 256 с.

7. Fliege J., Svaiter B. F. Steepest descent methods for multicriteria optimization. Mathematical Methods of Operations Research. 2000. Vol. 51. Issue 3. P. 479-494.

8. Zhu Q. J. Hamiltonian necessary conditions for a multiobjective optimal control problem with endpoint constraints. SIAM J. Control Optim. 2000. Vol. 39. No. 1. P. 97-112.

9. Ж Жуковский В. И., Чикрий А. А. Линейно-квадратичные дифференциальные игры. Киев: Наукова Думка, 1994. 320 с.

10. Ramos A. M., Glowinski R., Periaux J. Nash Equilibria for the Multi-Objective Control of Linear Partial Differential Equations. Journal of Optimization Theory and Applications. 2002. 112 (3). P. 457-498.

11. Ramos A. M., Glowinski R., Periaux J. Pointwise Control of the Burgers Equation and related Nash Equilibrium Problems: A Computational Approach. Journal of Optimization Theory and Applications. 2002. 112 (3). P. 499-516.

12. Lions J.-L. Controle de Pareto de Systemes Distribues: Le Cas stationnaire. Comptes Rendus de l'Academie des Sciences, Serie I. 1986. Vol. 302. P. 223-227.

13. Lions J.-L. Controle de Pareto de Systemes Distribues: Le Cas d'evolution. Comptes Rendus de l'Academie des Sciences, Serie I. 1986. Vol. 302. P. 413-417.

14. Nakoulima O., Omrane A., Velin J. On the Pareto Control and No-Regret Control for Distributed Systems with Incomplete Data. SIAM Journal on Control and Optimization. 2003. Vol. 42. No. 4. P. 1167-1184.

15. Бенсусан А., Лионс Ж.-Л., Темам Р. Методы декомпозиции, децентрализации, координации и их приложения. Методы вычислительной математики. Новосибирск: Наука, 1975. С. 144-274.

16. Lyashko S. I. Generalized optimal control of linear systems with distributed parameters. Boston-Dordrecht-London: Kluwer Academic Publishers, 2002.466p.

17. Ляшко С. І., Номіровський Д. А., Семенов В. В. Дослідження лінійних розподілених систем з узагальненим керуванням. Журнал обчислювальної та прикладної математики. 2004. № 2 (91). С. 31-45.

18. Семенов В. В., Семенова Н. В. О задаче векторного управления в гильбертовом пространстве. Кибернетика и системный анализ. 2005. № 2. С. 117-130.

19. Семенов В. В. Задача векторной оптимизации линейных распределенных систем с сингулярным управленим. Доповіді НАН України. 2004. № 10. С. 74-80.

20. Klyushin D. A., Lyashko S. I., Nomirovskii D. A., Petunin Yu. I., Semenov V. V. Generalized Solutions of Operator Equations and Extreme Elements. New YorkDordrecht-Heidelberg-London: Springer, 2012. 202+xxi p.

21. Чарин В. С. Линейные преобразования и выпуклые множества. Киев: Вища школа, 1978. 192 с.

22. Мордухович Б. Ш. Методы аппроксимаций в задачах оптимизации и управления. Москва: Наука, 1988. 359 с.

23. Дубовицкий А. Я., Милютин А. А. Задачи на экстремум при наличии ограничений. Докл. АН СССР. 1963. Т. 149. № 4. С. 759-762.

24. Kassay G., Radulescu V.D. Equilibrium Problems and Applications. London: Academic Press, 2019. 419 p.

25. Lyashko S. I., Semenov V. V. A New Two-Step Proximal Algorithm of Solving the Problem of Equilibrium Programming. In: Goldengorin B. (ed.) Optimization and Its Applications in Control and Data Sciences. Springer Optimization and Its Applications, 115, Springer, Cham, 2016. P. 315-325. 
26. Tammer Chr. Approximate solutions of vector-valued control-approximation problems. Studies in Locational Analysis. 1996. 10. P. 151-162.

27. Finet C. Perturbed minimization principles in partially ordered Banach spaces. Institut de Mathematique et d'Informatique, Universite de Mons-Hainaut. Preprint 2 (2000). P.1-16.

28. Михалевич В. С., Гупал А. М., Норкин В. И. Методы невыпуклой оптимизации. Москва: Наука, 1987. 279 с.

Надійшла: 13.10.2020 / Прийнята: 15.12.2020

\section{МЕТОДЫ ДЛЯ ЗАДАЧ ВЕКТОРНОГО ОБОБЩЕННОГО ОПТИМАЛЬНОГО УПРАВЛЕНИЯ СИСТЕМАМИ С РАСПРЕДЕЛЕННЫМИ ПАРАМЕТРАМИ}

\section{О. С. ХАрьков, Я. И. ВеДЕЛЬ, В. В. СЕмёНОВ}

Факультет компьютерных наук и кибернетики, Киевский национальный университет имени Тараса Шевченко, Киев, Украина,

E-mail: olehharek@gmail.com, yana.vedel@gmail.com, volodya.semenov@gmail.com

АннотАция. В работе развита теория существования и необходимых условий оптимальности для задач оптимального управления с векторным критерием качества системами с распределенными параметрами и обобщенными воздействиями. Исследовано понятие $(K, e, \varepsilon)$-аппроксимационной эффективности. Доказаны необходимые условия $(K, e, \varepsilon)$-аппроксимационной эффективности допустимых управлений в виде вариационных включений. Предложены методы решения задач векторной оптимизации линейных распределенных систем с обобщенным управлением. Доказана сходимость алгоритмов с погрешностями.

КЛЮчЕВЫЕ словА: векторная оптимизация, система с распределенными параметрами, обобщенное управление, условия оптимальности, алгоритм, сходимость. 